\title{
DOCUMENTATION
}

\section{CONTROLE ENDOCRINIEN ET NERVEUX DE L'OVULATION CHEZ LA POULE. FACTEURS INTERNES}

PAR

\author{
L. LACASSAGNE
}

Station de Recherches Avicoles, C.N.R.Z., Jouy-en-Josas

\section{PLAN DU MEMOIRE}

\section{- INTRODUCTION.}

\section{A. - FACTEURS HORMONAUX.}

I. - Rôle des gonadostimulines.

a) Action de F.S.H.

b) Action de L.H.

Io Réponse de l'ovaire à une injection de L.H.

- chez les poules normales.

- chez les animaux préalablement traités avec P.M.S.

$2^{\circ}$ Détermination du temps de latence séparant l'injection de $\mathrm{L}$.H. de la réponse du follicule ovarien.

$3^{\circ}$ Sensibilité du follicule en fonction de son degré d'évolution.

II. - Ovulations induites à l'aide de la progestérone.

III. - Limites de l'induction.

IV. - Ovulations induites par l'acétate de désoxycorticostérone et la testostérone.

V. - Action des œestrogènes.

B. - FACTEURS NERVEUX.

I. - Action des barbiturates.

II. - Action des agents sympatholytiques et parasympatholytiques.

III. - Importance de 1'heure d'injection.

IV. - Ponte et ovulation.

C. - ORIGINE DES SERIES.

I. - Hypothèse de FraPS.

II. - Hypothèse de Bastran et ZaRRow. 


\section{INTRODUCTION}

Une poule ayant une production régulière pond quotidiennement un œuf durant 2, 3 ou plusieurs jours successifs puis s'arrête généralement durant une journée pour recommencer sa ponte durant 2,3 ou plusieurs jours. Font partie d'une même série tous les œufs pondus sans interruption entre deux périodes de repos. Chaque ouf d'une série est pondu un peu plus tard que l'œuf précédent, le retard ainsi pris étant essentiellement fonction de la longueur de la série. En moyenne le retard décroît lorsque la longueur de la série croît.

Chez une poule pondant régulièrement l'heure d'ovulation de chaque ovocyte peut être déduite de l'heure de ponte de l'œuf correspondant à condition de connaître la longueur de la série. D'où application de la même définition de la série aux ovulations successives d'ovocytes. Chaque ovulation d'ovocyte intra série se produit donc comme pour les oufs avec un certain retard d'un jour sur l'autre.

Adoptant la terminologie de FraPS, nous appellerons $\mathrm{CI}$ le premier follicule d'une série, $\mathrm{C}_{2}$ le deuxième, etc., jusqu'à $\mathrm{CN}_{\mathrm{N}}$; le follicule $\mathrm{Cs}$ étant un follicule indéterminé de la série autre que $\mathrm{Cr}$.

La plupart des travaux ayant pour objet l'étude de l'ovulation ont été effectués sur des animaux spécialement choisis pour la régularité de leur série et maintenus dans des conditions d'élevage telles que la ponte de chaque œuf de la série se fasse à une heure constante d'une série sur l'autre et déterminée à l'avance, permettant d'évaluer l'induction ou l'inhibition d'une ovulation en fonction de l'heure présumée de l'ovulation naturelle.

\section{A. - FACTEURS HORMONAUX}

\section{I. - Rôle des gonadostimulines.}

\section{a) Action de F.S.H.}

Cette gonadotrophine joue un rôle essentiel dans la stimulation de l'ovogenèse mais nous ne rapporterons ici que les faits se rapportant au mécanisme de l'ovulation.

Le sérum de jument gravide, administré en injections sous-cutanées à la dose de 100 à 200 unités rat durant une dizaine de jours amène un développement remarquable de la grappe ovarienne mais provoque un arrêt total de la ponte au bout d'un jour ou deux (BATES, LAHR et RiDdLe I935, Fraps et RILEY, I942). L'énorme développement de la masse folliculaire ainsi obtenue serait en partie dû à la cessation de la ponte. A des doses inférieures à celles rapportées plus haut les injections de P.M.S. stimulent l'ovogenèse sans arrêter l'ovulation.

NALBANDOV et CARD I946, reprenant ces études à l'aide de F.S.H. (légèrement contaminée de L.H.) aboutissent, dans l'ensemble, aux mêmes résultats avec des doses totales de 400 à 500 unités rat injectées au cours d'une periode de I2 jours. L'analyse plus précise de la réponse ovarienne montre que l'action de F.S. H. diffère suivant la capacité de ponte de l'animal traité au moment du traitement.

- chez la poule en ponte dont l'ovaire contient normalement 2 à 4 follicules de taille ovulable ou subovulable des injections répétées de F.S.H. provoquent la cessation de la ponte et la maturation de nombreux follicules 
capables d'ovuler par administration de L. H. La cessation de la ponte serait due à la croissance brusque de ces gros follicules amenant un déséquilibre hormonal (Nalbandov et CARD, I946).

- chez les poules mauvaises pondeuses (environ un œuf tous les 4 jours) il n'existe normalement pas de séries graduées de follicules comme chez les bonnes pondeuses. L'ovaire comporte un gros follicule unique, seul appelé à atteindre une taille ovulable. Après l'ovulation de cet ovocyte il y a cessation de la ponte durant plusieurs jours jusqu'à ce que le follicule suivant atteigne une taille ovulable.

Injectée à ces animaux F.S.H., seule, est capable de provoquer une accélération de la ponte se traduisant par quelques ovulations. Peu d'ovulations étant obtenues après injection de L.H., il semble que dans ce cas il n'y ait pas de grossissement en masse des follicules pour atteindre la taille ovulable, la quantité de F.S.H. injectée étant suffisante pour établir une hiérarchie de follicule similaire aux séries graduées trouvées chez les bonnes pondeuses. Ce qui expliquerait qu'il n'y ait pas de déséquilibre endocrinien après le traitement (Nalbandov et CARD, I946).

- chez les poules non en ponte dont l'ovaire comporte des follicules très petits (tous inférieurs à I $\mathrm{mm}$ de diamètre) les injections de F.S.H. ne provoquent pas la ponte comme dans le cas précédent mais provoquent la maturation d'environ 4 follicules qui peuvent être amenés à ovuler par injection de L.H.. F.S.H. agit ici sur de nombreux follicules de même taille les forçant à croître simultanément, un grand nombre de follicules pouvant atteindre une taille ovulable en même temps. NALBANDOv et CARD I 946 avancent que cette masse de follicules démarrés serait endocrinologiquement assez puissante pour renverser 1'équilibre hormonal et empêcher L.H. endogène d'agir.

Il est intéressant de noter que l'effet d'un traitement au sérum de jument gravide diffère suivant le mode d'injection. Injecté par voie intraveineuse le P.M.S. provoque un avancement de l'heure d'ovulation.

\section{b) Action de L.H.}

Il ressort des expériences de FraPS, FEVOLD et NEHER I947, que parmi les différents extraits d'hypophyse de coq séparés selon la 'méthode de FEvoLD, I943, seule la fraction lutéinisante est spécifique de l'ovulation. Injectée par voie intraveineuse à la dose de o,OOI mg par poule I4 heures avant l'ovulation présumée de $\mathrm{C}_{\mathrm{I}}$, cette fraction lutéinisante provoque l'ovulation prématurée de ce follicule chez 50 p. Ioo des oiseaux traités. La fraction F.S.H. produit le même effet à la dose de $0,5 \mathrm{mg}$ par poule mais une telle quantité d'extrait contient vraisemblablement assez de L.H. pour conduire aux mêmes résultats.

L'action de L.H. est double : cette hormone provoque d'une part, la désagrégation rapide des parois de la vésicule germinale et l'expulsion du premier globule polaire dans les quatre heures qui suivent soit l'injection d'extrait d'hypophyse de poulet soit la libération normale de L.H. (OLSEN, Fraps et NEHER, 1947) et, d'autre part, la déhiscence du follicule ovarien 7 à 8 heures après le traitement ou la libération naturelle de L.H. 
Io Réponse de l'ovaire à une injection de préparation lutéinisante.

- chez les poules normales, non prétraitées au sérum de jument gravide une injection intraveineuse d'hormone lutéinisante peut induire l'ovulation du follicule mûr et de lui seul, jusqu'à I7 heures avant le temps escompté de lovulation naturelle.

\section{TABLEAU I}

Ovulation d'un seul follicule après injection intraveineuse de préparation d'hormone lutéinisante chez la poule.

\begin{tabular}{|c|c|c|c|c|c|c|}
\hline \multirow[b]{2}{*}{ Préparation } & \multirow[b]{2}{*}{ Unités (1) } & \multirow{2}{*}{$\begin{array}{c}\text { Heures } \\
\text { d'injection }\end{array}$} & \multicolumn{2}{|c|}{ Nombre poules } & \multicolumn{2}{|c|}{ Prématurité estimée } \\
\hline & & & Traitées & Ovulant & $\begin{array}{c}\text { Moyenne } \\
\text { (heure) }\end{array}$ & $\begin{array}{l}\text { Limite } \\
\text { (heure) }\end{array}$ \\
\hline $\begin{array}{l}\text { Préparation lutéinisante } \\
\text { dérivée d'hypophyse de } \\
\text { cheval contenant aussi } \\
\text { F. S. IH. en quantité } \\
\text { considérable. }\end{array}$ & $\begin{array}{r}20 \\
20 \\
20 \\
8 \\
4\end{array}$ & $\begin{array}{l}22 \mathrm{~h} 45 \\
\text { I9 } \mathrm{h} 30 \\
\text { I } 6 \mathrm{~h} \text { 00 } \\
\text { I6 h } 00 \\
\text { I6 h } 00\end{array}$ & $\begin{array}{l}7 \\
3 \\
3 \\
4 \\
3\end{array}$ & $\begin{array}{l}7 \\
3 \\
3 \\
4 \\
3\end{array}$ & $\begin{array}{r}3,48 \\
8,40 \\
11,20 \\
10,30 \\
10,30\end{array}$ & $\begin{array}{r}2,3-4, \mathrm{I} \\
8,3-8,5 \\
\text { I0,8-I I,7 } \\
\text { I0,8-I I ,7 } \\
9,7-10,8\end{array}$ \\
\hline
\end{tabular}

(1) Déterminées par l'accroissement en poids de vésicules séminales de rat suivant la méthode décrite par FEvolD H. L. Endocrinology I939, 24; 438. Doses de L. H. prédéterminées par les auteurs mais qui ne correspondent pas forcément à la dose optimum.

D'après Fraps, OLSEN et NeHER, 1942, Proc. Soc. Exp. Biol. Med., 50 ; 308 (partiel et modifié.)

Mais une préparation séchée d'hypophyse antérieure de coq Rhode Island injectée par voie intramusculaire à des poules après ovulation du follicule $\mathrm{C}_{I}$ et à des temps variables de 1'heure présumée de l'ovulation du follicule $\mathrm{C}_{2}$ supprime ou retarde un certain nombre de ces ovulations (FRAPS et FEVOLD, I955). Les auteurs rapportent l'action de cet extrait à son contenu en L.H. et F.S.H. d'après les résultats groupés au tableau II. Dans ce tableau deux sortes de mesures servent de critère de réponse. La première série de données représente simplement le nombre d'ovulations supprimées, sans égard au destin du follicule dont l'ovulation a été inhibée. La seconde mentionne le nombre d'ovulations qui auraient dî̀ se produire à ro $h$ 30 le lendemain du traitement et qui se trouvent simplement retardées jusqu'à 6 heures le jour suivant. Dans ce type de retard, le follicule dont l'ovulation a été retardée se comporte comme le follicule Cr d'une série débutant normalement. Une telle ovulation est reportée au tableau sous la dénomination " retard $\mathrm{CI}_{\mathrm{I}}$ ", la colonne suivante exprimant le rapport $\mathrm{R} / \mathrm{S}$ (retardées sur supprimées) exprimé en pour cent. Il semble que l'action de deux milligrammes de F.S.H. doive, comme plus haut, se ramener à l'action de L. H. inclue dans les impuretés de cette fraction de F.S.H. et Fraps et Fevoln concluent que F.S.H. en elle-même n'a petı ou pas d'action dans le retard de l'ovulation $\mathrm{C}_{2}$ mais qu'elle accroît le pouvoir de L.H. dans ce sens.

- chez les poules préalablement traitées au sérum de jument gravide l'injection d'une préparation d'hypophyse de cheval provoque l'ovulation simultanée de nombreux follicules à partir de la grappe ovarienne (FraPs et RILEY, I942). 
TABLEAU II

Nombre d'ovulations C2 retardées par injection intramusculaire, effectuée 17 h. 30 avant l'heure d'ovulation attendue, de L.H. et de F.S.H. soit sous forme séparée soit sous forme conjuguée.

\begin{tabular}{|c|c|c|c|c|c|}
\hline $\begin{array}{l}\text { Doses en mg } \\
\text { de LH }\end{array}$ & $\begin{array}{l}\text { Doses en } \mathrm{m} g \\
\text { de FSH }\end{array}$ & $\begin{array}{c}\text { Nombre } \\
\text { d'animaux traités }\end{array}$ & $\begin{array}{l}\text { Nombre d'ovula- } \\
\text { tions suppfimées }\end{array}$ & $\begin{array}{l}\text { Nombre } \\
\text { de retard Ci }\end{array}$ & $\begin{array}{c}\text { Rapport } \mathrm{R} / \mathrm{S} \\
\text { en } \%\end{array}$ \\
\hline 0,005 & - & I I & 2 & 2 & 100 \\
\hline $0,0 \mathrm{I}$ & $\ldots$ & II & 4 & 2 & 80 \\
\hline 0,02 & - & I 2 & 7 & 4 & 57 \\
\hline 0,04 & - & I4 & 6 & 5 & 83 \\
\hline - & 1,0 & 9 & I & 0 & - \\
\hline - & 2,0 & $2 \mathrm{I}$ & 7 & 6 & 86 \\
\hline 0,02 & 0.5 & 14 & 8 & 7 & 88 \\
\hline 0,02 & $\mathrm{I}, \mathrm{O}$ & 27 & $2 \mathrm{I}$ & 16 & 76 \\
\hline 0,02 & 2,0 & 14 & I3 & I 3 & 100 \\
\hline
\end{tabular}

D'après Fraps et Ftvold, Proc. Soc. Exp. Biol. Med., 90, 442, 1955 .

\section{TABLEAU III}

Ovulations suivant une seule injection intraveineuse de L.H. chez des poules prétraitées au sérum de jument gravide et absence d'ovulations chez les poules recevant le sérum de jument gravide seulement.

\begin{tabular}{|c|c|c|c|c|c|c|c|c|}
\hline \multicolumn{3}{|c|}{$\begin{array}{l}\text { Prétraitement au sérum de } \\
\text { jument gravide }\end{array}$} & \multirow{2}{*}{$\begin{array}{c}\text { Unités } \\
\text { de LH } \\
\text { injectées (1) }\end{array}$} & \multicolumn{3}{|c|}{ Poules ovulant } & \multicolumn{2}{|c|}{ Orulations } \\
\hline $\begin{array}{l}\text { Nombre } \\
\text { poules }\end{array}$ & $\begin{array}{l}\text { Doses quoti- } \\
\text { diennes unités } \\
\text { rat }\end{array}$ & Jours & & \begin{tabular}{|} 
Nombre \\
$24 \mathrm{~h}$ \\
avant LII
\end{tabular} & $\begin{array}{c}\text { Après ad } \\
\text { tion } \\
\text { Nombre }\end{array}$ & $\frac{H}{\%}$. & Total & $\begin{array}{c}\text { Par poule } \\
\text { ovulant }\end{array}$ \\
\hline I6 & IOO & 8-I0 & 20 & 0 & I 6 & 100 & 56 & 3,50 \\
\hline 16 & 200 & 10 & 20 & 0 & 13 & $8 \mathrm{I}$ & 4.3 & $3,3 I$ \\
\hline 4 & $100-200$ & II & 0 & o & 0 & - & 0 & 0 \\
\hline
\end{tabular}

(1) Déterminé par l'accroissement en poids de vésicules séminales de rat suivant la méthode décrite par FEvold H. L., Endocrinology, 1939, 24; 438. Doses de L. H. prédéterminées par les auteurs mais qui ne correspondent pas forcément à la dose optimum.

D'après Fraps R. M. et G. M. Riley, 1942. Proc. Soc. Exp. Biol. Med. 49 ; 253.

Comme nous l'avons vu au paragraphe précédent une injection de $\mathrm{L}_{\text {. }} \mathrm{H}$. à des poules prétraitées avec F.S.H. provoque tune réponse semblable (NALBANDOV et CARD, I946).

$2^{\circ}$ Détermination du temps de latence séparant l'injection de L.H. de la réponse du follicule ovarien.

La connaissance de ce temps de latence est fondamentale dans la détermination de l'heure de sécrétion naturelle de L.H. à partir de 1'heure d'ovulation et même de 1'heure de ponte chez une poule pondant régulièrement.

- Détermination indirecte. Le temps séparant une injection de L.H. de l'ovulation consécutive a pour la première fois été évalué chez đes animaux en ponte par FRAPS, RILEY et OLSEN, I942, à l'aide de la méthode mise au 
point par WARREN et ScotT, I935. Les résultats obtenus sont résumés dans le tableau et le graphique ci-dessous.

TABLEAU IV

Heure d'ounlation après injection intraveineuse d'une préparation de $L . H$. à 20 unités par poule, chez des poules White Leghorn prétraitées avec du sérum de jument gravide à 100 unités-rat par jour durant 8 jours.

\begin{tabular}{|c|c|c|c|c|c|}
\hline \multirow{3}{*}{$\begin{array}{c}\text { Nombre de } \\
\text { poules }\end{array}$} & \multirow{3}{*}{$\begin{array}{c}\text { Heure } \\
\text { d'injection }\end{array}$} & \multirow{3}{*}{$\begin{array}{c}\text { Temps écoulé injec- } \\
\text { tion à autopsie en } \\
\text { heures }\end{array}$} & \multirow{3}{*}{$\begin{array}{l}\text { Ovulations par } \\
\text { poule ovulant }\end{array}$} & \multicolumn{2}{|c|}{ Temps écoulé } \\
\hline & & & & \multicolumn{2}{|c|}{ Injection à ovulation } \\
\hline & & & & $\begin{array}{c}\text { Moyenne en } \\
\text { heures }\end{array}$ & $\begin{array}{l}\text { Temps limite } \\
\text { en heures }\end{array}$ \\
\hline $\begin{array}{l}5 \ldots \ldots \ldots \\
4 \ldots \ldots \ldots \ldots \\
3 \ldots \ldots \ldots \ldots \\
3 \ldots \ldots \ldots\end{array}$ & 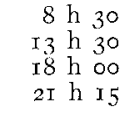 & $\begin{array}{l}7,2 \\
7, \mathrm{I} \\
7, \mathrm{I} \\
7, \mathrm{I}\end{array}$ & $\begin{array}{l}1,5 \\
2,3 \\
3,3 \\
2.7\end{array}$ & $\begin{array}{l}7,0 \\
6,7 \\
6,9 \\
6,6\end{array}$ & $\begin{array}{l}6,6-7,2 \\
6,3-7,1 \\
6,6-7,1 \\
6,1-7,0\end{array}$ \\
\hline
\end{tabular}

D'après Fraps, Rilex et Olsen, 1942, Proc. Soc. Exp. Biol. and Med., 50, 3 r3.

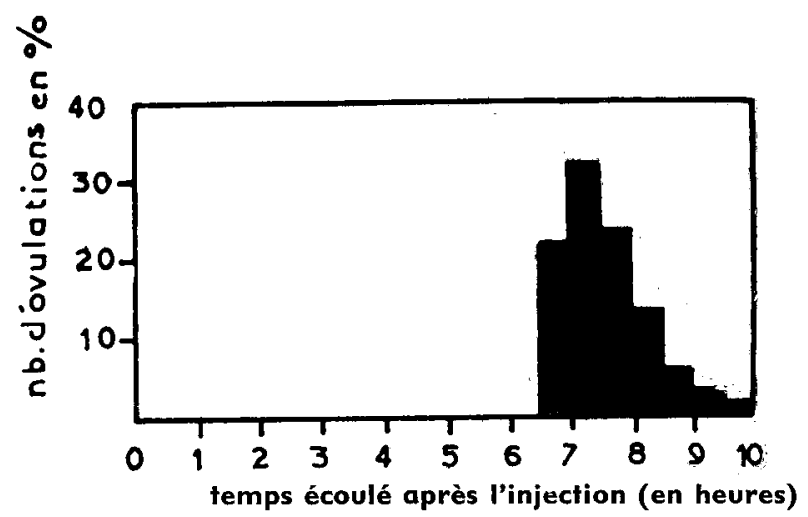

GRAPHIQUE I. - Distribution des ovulations induites par L. H. chez des poules non prétraitées avec F.S.H., d'après Fraps, Riley et Olsen. I942, Proc. Soc. Exp., Biol. Med., 50 ; 3 I3 (modifié).

Le temps écoulé entre l'injection et l'ovulation est en moyenne significativement plus court et de variabilité moins grande chez les poules prétraitées que chez les poules non prétraitées, phénomène sans doute dû à une plus grande maturité des follicules dans le premier cas.

Détermination directe. - Il ressort des expériences de Ro'THCHILD et FRAPS I949 $a$, que l'hypophyse n'est pas nécessaire à l'ovulation dans un intervalle de 4 à 6 heures avant celle-ci. La dose de L.H. nécessaire à la déhiscence du follicule semble donc libérée en totalité 6 heures avant 1'ovulation. Ces chiffres amènent les auteurs à penser que les mécanismes présidant à la libération de L.H. ont généralement lieu durant les heures d'obscurité ou encore durant les périodes où l'animal ne prend aucun exercice.

Comme nous le verrons plus loin, Van Tienhoven, Nalbandov et NorTon I954, en se fondant sur des expériences effectuées à l'aide de la dibénamine, suggèrent que le stimulus conduisant à la libération de L.H. prend naissance 8 à I 4 heures avant l'ovulation. Ces chiffres ne sont pas en contra- 
diction avec les précédents si on admet que la libération de la dose de L.H. nécessaire à l'ovulation s'étale sur une assez grande période.

\section{$3^{0}$ Sensibilité du follicule en fonction de son degré d'évolution.}

FRAPS et DURY I942, rapportent que le premier follicule (CI) d'une série est 20 fois plus sensible à l'ovulation par injection d'extrait d'hypophyse de mouton que ne le sont les follicules suivants à des temps comparables avant leur ovulation normale. Reprenant cette étude en détail BASTIAN et ZARROw I955, ont élaboré une courbe de sensibilité des follicules $\mathrm{C}_{1}$ et $\mathrm{C}_{2}$ à des injections de $\mathrm{L}_{1} . \mathrm{H}_{\mathrm{H}}$. effectuées à différents stades de leur maturité. Le deuxième follicule de la série $(\mathrm{C} 2)$ montre une plus grande variabilité de réponse aux injections de gonadotrophine. Ceci semble dû à la moins grande précision dans la prédiction de l'ovulation à venir et au fait que chaque follicule $\mathrm{C}_{2}$ possède un seuil légèrement différent suivant le moment du jour ou de la nuit qui précède son ovulation. Ces considérations peuvent expliquer que les chiffres expérimentaux sur le follicule $\mathrm{C}_{2}$ sont plus variables que pour le follicule $\mathrm{Cr}$

Ces auteurs rapportent qu'une injection de L.H. destinée à provoquer une ovulation prématurée du premier follicule, montre qu'un état de haute sensibilité est atteint environ 24 heures avant le moment de l'ovulation normale. Une dose équivalente à Io $\mathrm{mg}\left({ }^{1}\right)$ de L.H. ne provoque pas l'ovulation du follicule $C_{I}$, quand elle est injectée 40 à 42 heures avant l'ovulation. A $36-38$ heures, cependant, $5 \mathrm{mg}$ de $\mathrm{I}_{4} . \mathrm{H}$. induisent l'ovulation et cette dose est réduite à $I, 5 \mathrm{mg}$ à $26-28$ heures, commencement de la période de sensibilité maximum. Cela est parfaitement en accord avec les résultats de Fraps I946, qui conclut que le fait que le follicule Ci n'ovule pas plus tôt doit être le résultat d'un "manque d'excrétion d'hormone d'ovulation ". Il est également possible de conclure qu'il y a une secrétion de l'hormone d'ovulation dans la nuit qui précède l'ovulation mais à une dose telle qu'elle est incapable d'induire la rupture du follicule dont la maturation n'est pas assez avancée. Cela peut expliquer la présence d'une période de plus grande sensibilité 24 heures avant.

Le follicule $\mathrm{C}_{2}$ est moins sensible que le follicule $\mathrm{C}_{\mathrm{a}}$ à une injection de $\mathrm{L}_{\text {. }} \mathrm{H}$ : 20 heures avant l'ovulation prévue, le follicule $C_{I}$ présente une sensibilité approximative de $0,5 \mathrm{mg}$ de L.H. contre $4 \mathrm{mg}$ pour le follicule $\mathrm{C} 2$. Ce rapport (8 à I) est moindre que la différence de 20 fois rapportée plus tôt par Fraps I946.

Cette inégalité de réponse des follicules $\mathrm{C}_{\mathrm{I}}$ et $\mathrm{C}_{2}$ amène les auteurs à penser que les follicules successifs d'une série sont ovulés à des stades de maturité moindre. Cependant, si à l'aide des données de Bastias et ZaRkow le calcul de la sensibilité est effectué non en fonction de l'heure de l'ovulation prévue mais en fonction du temps séparant l'injection de l'ovulation précédente, il est possible de constater que la sensibilité des follicules $C_{I}$ et $\mathrm{C}_{2}$ à des injections de L.H. est approximativement la même à des temps comparables. Ces faits en accord avec les observations non publiées de FrAPS, ROTHCHILD et NEHER et auxquelles il est fait allusion dans le rapport de FraPS I955, sont à la base, comme nous le verrons plus loin, de l'élaboration des hypothèses tendant à expliquer la constitution des séries.

(i) (Exprimés en préparation standard des laboratoires ARMour de Chicago). 


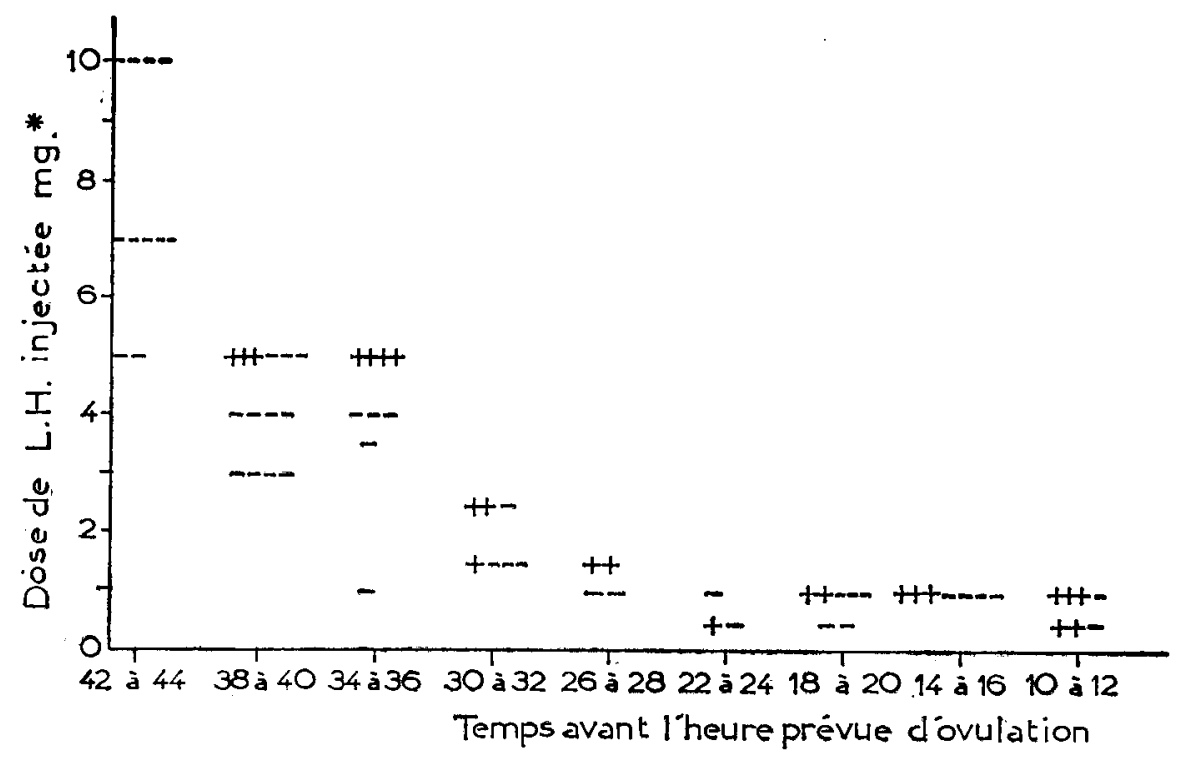

GRAPHIQUE II. - Ovulation induite du premier follicule de la série, chaque signe (+) représente une ovulation induite à l'aide de L. H. Chaque signe $(-)$ représente un échec dans l'induction de l'ovulation prématurée.

* (Exprimés en préparation standard des laboratoires ARMOUR, Chicago), d'après BASTIAN et Zarrow., r955 Poul. Sci., 34, 776 (modifié).

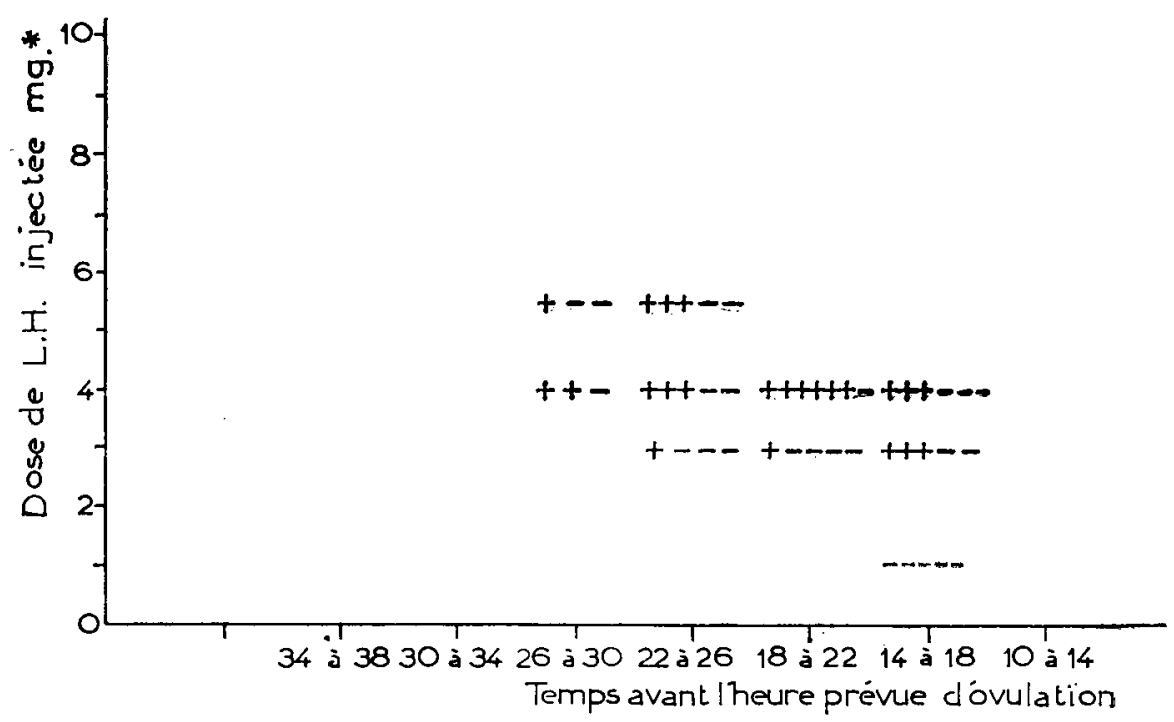

GRAPHIQUE III. - Ovulation induite des follicules suivants de la série. Chaque signe (+) représente une ovulation induite à l'aide de $\mathrm{L}$. H. Chaque signe $(-)$ représente un échec dans l'induction de l'ovulation prématurée.

* (Exprimés en préparation standard des laboratoires ARMour, Chicago), d'après Bastian et Zarrow., r955 Poul. Sci., 34; 776 (modifié). 


\section{II. - Ovulations induites à l'aide de la progestérone.}

L'action de la progestérone sur le follicule ovarien dépend essentiellement de la voie d'administration, de l'heure d'injection en fonction de l'heure d'ovulation prévue et de la place du follicule dans la série. C'est ainsi que les follicules $\mathrm{C}_{I}$ et $\mathrm{Cs}_{S}$ répondent de façon différente. En injection intraveineuse la progestérone provoque l'ovulation du follicule Cr mais non des follicules Cs. Sous l'action d'une piqûre sous-cutanée, les follicules $\mathrm{Cr}$ et Cs répondent tous det1x, avec cependant une sensibilité plus grande pour CI (FraPs et DURY I943).

\section{TABLEAU V}

Aptitude de la progestérone, injectée à 16 heures,

à induire prématurément l'ovulation des follicules ovariens de la poule au moins 6 heures à l'avance.

\begin{tabular}{|c|c|c|c|c|c|c|c|}
\hline \multicolumn{2}{|c|}{ Injection } & \multicolumn{3}{|c|}{ Poules avec follicules $C_{1}$} & \multicolumn{3}{|c|}{ Poules avec follicules $\mathrm{Cs}$} \\
\hline \multirow{2}{*}{ Voie } & \multirow{2}{*}{ Doses mg. } & \multirow{2}{*}{ Injectées } & \multicolumn{2}{|c|}{ Ayant ovulé } & \multirow{2}{*}{ Injectées } & \multicolumn{2}{|c|}{ Ayant ovulé } \\
\hline & & & $\mathrm{Nb}$ & $\%$ & & $\mathrm{Nb}$ & $\%$ \\
\hline $\begin{array}{c}\text { Intra vein. } \\
, \\
" \\
" \\
" \\
" \\
" \\
"\end{array}$ & $\begin{array}{c}0,01 \\
0,02 \\
0,05 \\
0,20 \\
0,50 \\
\mathrm{I}, 00 \\
\mathrm{x}, 5-5,0 \\
\mathrm{I0,0} \\
\mathrm{I} 5,0-40,0\end{array}$ & $\begin{array}{r}- \\
9 \\
13 \\
27 \\
9 \\
10 \\
17 \\
\end{array}$ & $\begin{array}{r}- \\
\text { I } \\
4 \\
19 \\
8 \\
9 \\
10 \\
-\end{array}$ & $\begin{array}{l}- \\
\mathrm{II}, \mathrm{I} \\
30,8 \\
70,4 \\
88,9 \\
90,0 \\
- \\
58,8 \\
-\end{array}$ & $\begin{array}{r}10 \\
20 \\
18 \\
11 \\
6 \\
7 \\
8 \\
7 \\
16\end{array}$ & $\begin{array}{l}0 \\
0 \\
0 \\
0 \\
0 \\
0 \\
0 \\
I \\
I\end{array}$ & $\begin{array}{c}0 \\
\circ \\
0 \\
0 \\
0 \\
0 \\
0 \\
14,3 \\
6,3\end{array}$ \\
\hline $\begin{array}{c}\text { Sous cutan. } \\
" \\
" \\
" \\
" \\
" \\
" \\
" \\
"\end{array}$ & $\begin{array}{l}0,05 \\
0,125 \\
0,25 \\
0,50 \\
1,0 \\
2,0 \\
3,0 \\
5,0 \\
10\end{array}$ & $\begin{array}{r}20 \\
21 \\
29 \\
25 \\
19 \\
11 \\
7 \\
70\end{array}$ & $\begin{array}{r}0 \\
4 \\
20 \\
19 \\
18 \\
10 \\
7 \\
9\end{array}$ & $\begin{array}{c}0 \\
19,0 \\
69,0 \\
76,0 \\
94,7 \\
90,9 \\
- \\
100,0 \\
90,0\end{array}$ & $\begin{array}{r}- \\
9 \\
8 \\
15 \\
14 \\
7 \\
13 \\
11 \\
10\end{array}$ & $\begin{array}{r}- \\
0 \\
0 \\
\text { II } \\
\text { I0 } \\
5 \\
10 \\
8 \\
5\end{array}$ & $\begin{array}{c}- \\
\circ \\
\circ \\
73,3 \\
71,4 \\
71,4 \\
76,9 \\
72,7 \\
50,0\end{array}$ \\
\hline
\end{tabular}

D’après Fraps R. M. et Abraham Dury, Proc. Soc. Exp. Biol. Med., 1943, 52, 346.

Les follicules s'individualisent également par leur temps de latence. La progestérone induit l'ovulation de $\mathrm{CI}$ entre 7 et 9 heures après l'injection, les follicules Cs présentent une réponse un peu plus tardive entre 8 et II heures après l'administration de progestérone, chaque injection étant faite à des moments identiques par rapport à 1'heure présumée de l'ovulation naturelle

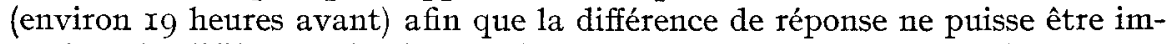
putée à la différence des intervalles compris entre l'injection et le moment de 1'ovulation présumée (FRAPs et DuRY I943).

Le défaut de réponse de $\mathrm{Cs}$ en injection intraveineuse peut s'expliquer par la rapidité avec laquelle la progestérone ainsi administrée disparaît de la circulation sanguine (EDGAR I953), ce qui sous-entend que pour agir sur Cs cette hormone doit agir plus longtemps sur le mécanisme présidant à la libération de L.H. endogène. Cette particularité, jointe à la moins grande 
sensibilité de $\mathrm{Cs}$ par rapport à $\mathrm{CI}_{\mathrm{I}}$ à une même dose de progestérone semble indiquer ici aussi que la "maturité " du follicule $\mathrm{Cr}$ se trouve plus avancée que celle du follicule $\mathrm{Cs}$ à des intervalles identiques avant l'heure présumée d'ovulation.

Un prétraitement avec du sérum de jument gravide change complètement la réponse des animaux aux injections de progestérone qui ne provoquent plus d'ovulation chez les animaux ainsi traités. RoTHCHID et FRAPS I949 $b$ ont analysé le mécanisme d'action de la progestérone sur l'ovaire afin d'expliquer ce manque de réponse chez les animaux traités avec le sérum de jument gravide.

Ces auteurs prélèvent des hypophyses antérieures de poules prétraitées au P.M.S. et constatent que la capacité de ces glandes à induire l'ovulation est bien inférieure à celle des poules non traitées. D'où l'hypothèse que la progestérone agirait par l'intermédiaire de l'hypophyse. Cette glande aurait normalement une réserve suffisante de $\mathrm{L} . \mathrm{H}$. pour répondre à la progestérone, réserve qui aurait disparue après traitement au sérum de jument gravide. A 1'appui de cette hypothèse, les auteurs montrent que la progestérone est incapable d'induire 1'ovulation soit des follicules CI soit des follicules Cs chez les animaux hypophysectomisés. L' hypophyse doit rester intacte de 2 à 4 heures après injection de progestérone pour qu'il soit possible d'enregistrer une réponse des follicules ovariens. Selon Van TIENHOVEN (I954) la durée minima de stimulation hypophysaire accompagnant l'injection de progestérone est de 1'ordre de I,30 heures. Van TIENHOVEN suggère que la stimulation de la pituitaire et la libération de L.H. ont lieu simultanément. S'il en est ainsi, l'intervalle entre le début de la libération de L.H. et l'ovulation est pratiquement identique à 1'intervalle entre l'injection de progestérone et l'ovulation soit 8 heures pour le follicule Cr. L'intervalle entre le moment où la concentration de L.H. est suffisante et l'ovulation est alors de 6,30 heures, chiffre à rapprocher du temps de latence d'une injection intraveineuse de L.H. destinée à induire une ovulation prématurée, qui est de 6,30 à $\delta$ heures (Fraps, RILEy et OlsEN, I942).

Il semble que les doses de progestérone nécessaires à l'induction de l'ovulation soient variables en fonction de facteurs nerveux. HUSTON et NALBANDOV (I953) réussissaient à provoquer l'ovulation au moyen de la progestérone chez des poules dont la sécrétion de L.H. avait été bloquée par la présence d'un fil chirurgical dans le magnum. Les doses nécessaires furent de 0,3 à $6 \mathrm{mg}$., doses apparemment plus élevées que celles trouvées par RoTHCHILD et FraPS (I949). La progestérone agit différemment suivant la dose employée.

L'effet inhibiteur de la progestérone, décrit chez les mammifères, a été démontré chez la poule. Chez ces dernières l'inhibition de l'ovulation accompagne l'injection de progestérone faite à des intervalles de 36 heures ou plus avant l'ovulation attendue. Aux mêmes doses, mais à des fréquences plus répétées que celles précédemment citées, la progestérone induit la mue. Après administration de $20 \mathrm{mg}$ de progestérone injectées lentement à des doses de $0,5 \mathrm{mg}$ au cours de plusieurs jours, la production cesse au bout de deux semaines et les poules muent rapidement. La diversité d'action suivant la dose a également été constatée chez d'autres espèces (DAUzIER, ORTAVANT, THIBAULT et WINTENBERGER, I953). Le même traitement effectuée en plein hiver n'est pas efficace et ne provoque pas une mue complète (SHAFFnER I955). 


\section{III. - Limites de l'induetion.}

L'induction d'ovulations à l'aide de L. H. ou de progestérone a cependant une limite qui diffère avec chaque poule, même chez des animaux dont les conditions physiologiques semblent à peu près identiques. C'est ainsi que NEHER et FRAPS, I950, induisent à l'aide d'extrait non fractionné d'hypophyse de coq séchée à l'air ou à l'aide de progestérone des ovulations additives à une série de deux œufs. Les injections furent effectuées à des intervalles de 24 , 26 ou 28 heures pour respecter les temps intra série, et à des heures telles, que 1'ovulation survenant 6 à 8 heures après, se trouve à sa place dans la série, le deuxième ouf étant induit, cela jusqu'à ce que l'ovaire ne réponde plus (voir tableau).

\section{TABLEAU VI}

Ponte des poules avant, durant et après l'administration de progestérone à la dose de 0,6 $\mathrm{mg}$ par animal et par jour, injectée à 8 heures.

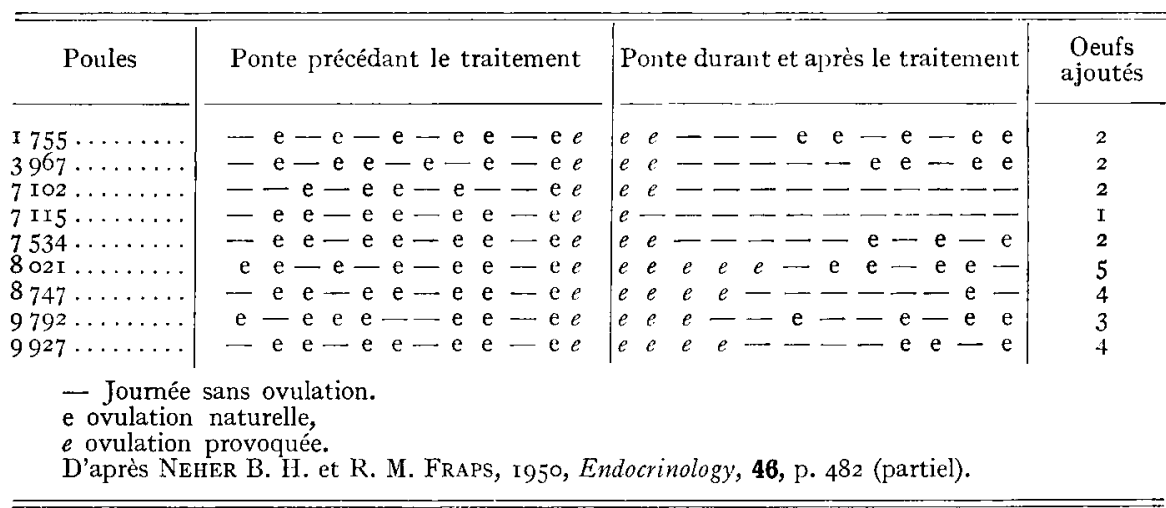

Le nombre d'œufs ainsi ajoutés à la série est résumé dans le tableau suivant.

\section{TABLEAU VII}

Oeuts ajoutés à une série anticipée de 2 xufs après injection de préparation d'hypophyse antérieure (A. P.) ou de progestérone (Pg.)

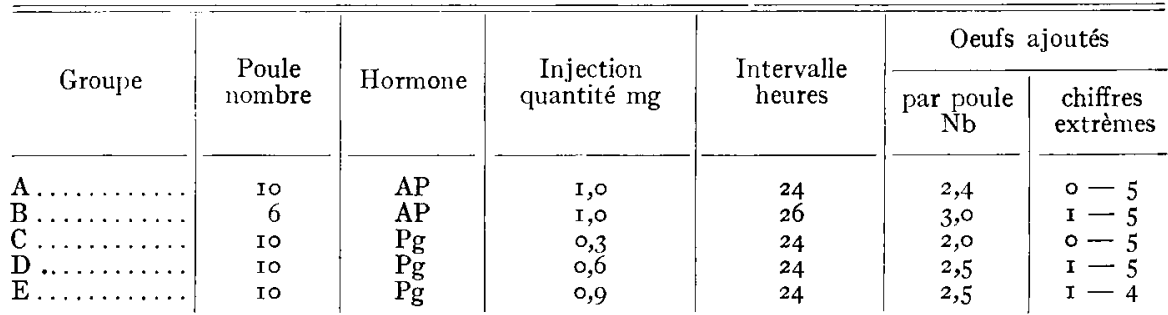

D'après Neher B. H. et R. M. FraPS, I950, Endocrinology, 46, 482 (modifié). 
Suivant l'animal considéré, des injections de L. H. ou de progestérone effectuées en temps voulu ajoutent donc I à 5 œuffs à une série normale de deux œufs.

NEHER et FRAPS, I950, citent également le cas intéressant d'une poule ayant pondu $\mathrm{I}_{3}$ œufs supplémentaires à une série de deux œufs. Comme le montre le tableau ci-dessous, le poids du vitellus va en décroissant dans la série, indiquant un déséquilibre entre la vitesse d'ovulation et celle de 1'ovogenèse, plus vraisemblablement de la vitellogenèse, qui fait intervenir une mobilisation considérable de matériaux.

\section{TABLEAU VIII}

Poids des ceufs et des jaunes du second auf d'une série normale et des 13 aufs successifs ajoutés expérimentalement à l'aide d'injection de progestérone (poule 2477).

\begin{tabular}{c|c|c|c}
\hline $\begin{array}{c}\text { Heure approximative } \\
\text { de la ponte }\end{array}$ & $\begin{array}{c}\text { Temps écoulé depuis la } \\
\text { ponte précédente. Heures }\end{array}$ & $\begin{array}{c}\text { Poids des ceufs } \\
\text { en grammes }\end{array}$ & $\begin{array}{c}\text { Poids du jaune en } \\
\text { grammes }\end{array}$ \\
\hline $\mathbf{1 2}$ & - & 55,0 & 15,7 \\
2 & 26 & 53,4 & 14,8 \\
6 & 28 & 54,5 & 14,5 \\
22 & 28 & 55,4 & 14,2 \\
3 & 29 & 56,2 & 14,7 \\
7 & 28 & 56,2 & 14,7 \\
11 & 28 & 53,5 & 14,0 \\
15 & 28 & 52,0 & 13,0 \\
19 & 28 & 51,4 & 13,3 \\
24 & 29 & 50,6 & 12,4 \\
5 & 29 & 50,6 & 12,3 \\
2 & 28 & 50,6 & $1 \mathrm{r}, 3$ \\
12 & 27 & 47,9 & 10,1
\end{tabular}

D'après Neher B. H. et Fraps R. M., 1950, Endocrinology, 46, 482.

L'injection d'extrait lutéinisant d'hypophyse de mouton à des doses submaximales provoque la régression des follicules intra série chez les poules qui n'ovulent pas. Le follicule $\mathrm{CI}_{\mathrm{I}}$ peut parfois régresser dans les mêmes conditions (Fraps et DURY, I942 a, BASTIAN et ZARRow, I955).Van TrEnhoven, r955, a récemment montré que l'interruption de la libération de L.H., induite par la progestérone, peut provoquer 1'atrésie du follicule ovulable. Ces expériences jointes à celles d'EVERETT et SAWYER, I953, soutiennent 1'hypothèse selon laquelle l'atrésie serait due à une stimulation partielle du follicule.

\section{IV. - Ovulations induites par l'acétate. de desoxycorticostérone et laltestostérone.}

L'acétate de désoxycorticostérone et la testostérone induisent également des ovulations prématurées chez la poule.

L'acétate de désoxycorticostérone paraît d'une efficacité comparable à celle de la progestérone. Pour les deux produits, l'intervalle séparant l'injection de l'ovulation est inférieur à 8 heures.

La testostérone a une action moindre que celle de la progestérone et de l'acétate de désoxycorticostérone : le temps s'écoulant entre l'injection et 1'ovulation est supérieur à 9 heures. 


\section{TABLEAU IX}

Ovulations de C1 induites par l'acétate de désoxycorticostérone. et la testostérone en injection aux doses de $1 \mathrm{mg}$ par animal.

\begin{tabular}{|c|c|c|c|}
\hline & \multirow{2}{*}{$\begin{array}{c}\text { Nombre de poules } \\
\text { traitées }\end{array}$} & \multicolumn{2}{|c|}{ Poules ayant ovulé } \\
\hline & & Nombre & $\%$ \\
\hline $\begin{array}{l}\text { Progestérone.................... } \\
\text { Acétate de desoxycorticostérone.. } \\
\text { Testostérone.................. }\end{array}$ & $\begin{array}{l}19 \\
17 \\
32\end{array}$ & $\begin{array}{l}\text { I8 } \\
\text { I5 } \\
\text { I3 }\end{array}$ & $\begin{array}{l}95 \\
87 \\
4 \mathrm{I}\end{array}$ \\
\hline
\end{tabular}

Ceci semble indiquer que progestérone et acétate de désoxycorticostérone peuvent agir directement dans le forçage de l'ovulation (1'acétate de désoxycorticostérone peut également être converti en progestérone "in vivo", ZARROW, HISAW et BRYANS, I950), alors que la testostérone subirait une conversion plus lente en substance active. La testostérone s'individualise également en ce que, dans certaines conditions, elle peut retarder l'ovulation plus que ne le fait le benzoate d'œstradiol.

\section{V. - Action des aestrogènes. I}

L'injection intramusculaire de I à 2,5 mg de benzoate d'œstradiol par poule, effectuée I $_{4}$ à 4 I heures avant l'ovulation attendue de Ci et II à 28 heures avant l'ovulation attendue de $\mathrm{C} 2$, donne les réstultats résumés au tableau suivant :

\section{TableaU $X$}

Pourcentage d'ovulations supprimées ou retardées après injection de benzoate d'cestradiol, en fonction de l'intervalle de temps séparant l'injection de l'heure présumée d'ovulation, chez des poules pondant deux aufs par série.

\begin{tabular}{|c|c|c|c|c|c|c|}
\hline \multirow{2}{*}{$\begin{array}{l}\text { Heures d'ovu- } \\
\text { lation attendue }\end{array}$} & \multirow{2}{*}{$\begin{array}{c}\text { Temps écoulé } \\
\text { entre l'injection } \\
\text { et l'heure d'ovu- } \\
\text { lation attendue } \\
\text { (heures) }\end{array}$} & \multirow{2}{*}{$\begin{array}{c}\text { Nombre de } \\
\text { poules traitées }\end{array}$} & \multicolumn{2}{|c|}{ Ovulations supprimées } & \multicolumn{2}{|c|}{$\begin{array}{l}\text { Ovulations retardées } \\
\text { d'un jour }\end{array}$} \\
\hline & & & $\mathrm{Nb}$ & $\%$ & $\mathrm{Nb}$ & $\%$ \\
\hline $\mathrm{C}_{1}$ à 6 heures: & $\begin{array}{c}\text { I } 4 \\
24 \\
4 \mathrm{I} \\
\text { I } 4 \text { à } 4 \mathrm{I}\end{array}$ & $\begin{array}{l}56 \\
\text { I6 } \\
\text { I6 } \\
88\end{array}$ & $\begin{array}{r}7 \\
3 \\
2 \\
\mathrm{I} 2\end{array}$ & $\begin{array}{l}\text { I3 } \\
\text { I9 } \\
\text { I3 } \\
\text { I4 }\end{array}$ & $\begin{array}{r}5 \\
2 \\
\mathbf{I} \\
8\end{array}$ & $\begin{array}{r}9 \\
\text { I3 } \\
6 \\
9\end{array}$ \\
\hline$\frac{\mathrm{C}_{2} \dot{\mathrm{a}}}{\text { Io heures 30 }}$ & 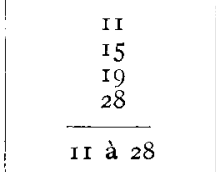 & $\begin{array}{l}13 \\
\text { r5 } \\
60 \\
31 \\
\text { I I9 }\end{array}$ & $\begin{array}{r}9 \\
7 \\
31 \\
19 \\
66\end{array}$ & $\begin{array}{l}69 \\
47 \\
52 \\
61 \\
-55\end{array}$ & $\begin{array}{r}6 \\
5 \\
28 \\
15 \\
54\end{array}$ & $\begin{array}{l}46 \\
33 \\
47 \\
48 \\
45\end{array}$ \\
\hline
\end{tabular}

Comme le montre l'examen du tableau les follicules $\mathrm{Cr}$ et $\mathrm{C}_{2}$ n'ont pas la 
même sensibilité : le follicule $\mathrm{C} 2$ est plus sensible au benzoate d'œstradiol que le follicule Cr. De plus, il s'avère que l'effet de l'œstradiol, dans la suppression ou le retard d'un jour de l'ovulation des follicules $\mathrm{Cr}$ et $\mathrm{C} 2$, est indépendant du temps écoulé entre l'injection et l'ovulation normalement attendue.

Les ovulations retardées des follicules $\mathrm{C}_{\mathrm{I}}$ ont lieu durant les heures normales de ponte 24 heures plus tard ou à ces mêmes heures multiples de 24 .

Les ovulations retardées $\mathrm{du}$ follicule $\mathrm{C}_{2}$ se produisent également aux heures d'ovulation c'est-à-dire de 6 heures à $I_{4}$ heures environ. La plupart des ovulations différées ont cependant lieu vers 6 heures indiquant que lorsque 1'ovulation $\mathrm{C}_{2}$ est retardée, son follicule se comporte comme un follicule $\mathrm{Cr}_{\mathrm{r}}$, le retard étant cependant de I9-20 heures et non pas de 24 heures comme c'est le cas pour $\mathrm{Cr}$.

Le dipropionate d'œstradiol et le diéthylstilbestrol ont une action comparable, mais plus marquée, à celle du benzoate d'œtradiol et leur effet différentiel sur $\mathrm{C}_{1}$ et $\mathrm{C}_{2}$ est à peu près du même ordre que celui fourni par une injection de 2 à $5 \mathrm{mg}$ de benzoate d'œstradiol par poule, injectés I 4 heures avant l'ovulation attendue de $\mathrm{C}_{\mathrm{I}}$ et $\mathrm{I} 9$ heures avant celle de $\mathrm{C}_{2}$.

\section{B. - FACTEURS NERVEUX DE L'OVULATION}

Aucun travail n'a été fait sur la physiologie des nerfs du système nerveux autonome innervant l'ovaire et l'oviducte. Quelques plexus nerveux décrits par MaUger (I94I) furent localisés par Sturkie et WeIss, STURkIE (I954), mais leur position et leur taille sont telles qu'il n'est possible de les stimuler chez la poule ouverte qu'après une manipulation considérable de viscères, ce qui introduit une cause d'erreur importante.

Aussi tous les travaux se rapportant à l'étude de ces facteurs nerveux font-ils appel à des constatations ou à des études indirectes (lésions, administration de barbiturates ou de sympathicomimétiques) dont voici l'essentiel :

Ro'THCHILD et FRAPS, I $949 a$, ont montré que le taux de I.H. nécessaire à l'ovulation était atteint 4 à 6 heures avant l'ovulation. Ainsi, les facteurs qui peuvent être mis en jeu soit dans la stimulation soit dans l'inhibition de L.H. doivent exercer leur effet plusieurs heures avant l'ovulation. Puisqu'il y a un intervalle relativement court entre l'oviposition et l'ovulation consécutive (de 30 à 60 minutes), il apparaît que les événements conduisant à 1'ovulation surviennent normalement alors que l'œuf précédant est encore dans la glande coquillère.

Huston et NALBANDOV, I953, ont prouvé que l'irritation de la lumière du magnum par 1'attache d'un fil chirurgical inhibe toute ovulation pendant une durée de ro à 30 jours en agissant spécifiquement sur le rejet de L.H sans interférence sur les autres hormones en provenance de l'hypophyse comme le montrent la taille et l'aspect complètement normaux de l'ovaire de l'oviducte et de la crête des poules ainsi traitées.

Les auteurs ont démontré que ce fil agissait spécifiquement sur le rejet de I.H. car une injection de o, I mg de L.H. à des poules ainsi traitées provoque l'ovulation d'un ovocyte. Ce fait et la constatation que la crête de la poule n'est pas atteinte, amènent les auteurs à la généralisation suivante : un certain taux de L. H. est sécrété constamment par l'hypophyse qui, périodiquement, relâche des quantités d'hormone plus fortes ou " ovulatory peaks " capables d'induire l'ovulation d'un ovocyte et inhibées par la présence d'un fil. 
De même la présence d'un œuf dans le magnum supprimerait neurologiquement ces " sommets ovulatoires " empêchant ainsi l'ovulation aussi longtemps qu'il y a un ouf dans cette partie de l'oviducte. Après le passage de l'œuf dans le magnum, le mécanisme inhibiteur de l'hypophyse disparaîtrait et I.H. serait sécrétée en quantité suffisante pour provoquer l'ovulation suivante.

Après 20 à 30 jours durant lesquels les ovocytes demeurent en état quiescent et non atrésique, la ponte reprend progressivement. L'oviducte est devenu insensible au stimulus du fil comme le montre le fait que l'introduction d'un nouveau fil après ce délai ne bloque plus la ponte.

Ces travaux ont été confirmés par Van TrenHoven, I953, qui note des différences quantitatives dans la suppression de la ponte suivant la région du magnum oì se trouvent implantées les boucles de fil. I'effet s'accroît avec la distance à mesure que l'on s'éloigne de l'infundibulum et est plus grand dans l'isthme que dans le magntum.

L'action de ces excitants n'est cependant pas aussi schématique qu'il vient d'être dit. Si l'excitation est forte : introduction dans le magnum de maillons de chaînette, etc., il peut y avoir au contraire superovulation et fomation d'œufs hardés ou ponte de jaunes simplement recouverts de blanc ou même de jaunes nus (Huston et Nalbandov, I953).

La présence d'une suture chirurgicale dans l'utérus donne naissance à la production d'œufs hardés chez plus de la moitié des oiseaux opérés, mais l'ovulation continue à un taux sensiblement normal. Le jaune ne peut être retenu dans l'oviducte assez longtemps pour que la formation de l'œuf puisse être achevée (SyKES, I935 b). L'ovulation est presque complètement inhibée chez chez les oiseaux dont la moelle épinière a été détruite. A l'autopsie les ovaires des animaux ainsi traités présentent un ou plusieurs follicules atrésiques parmi les plus gros follicules de l'ovaire. Certains cependant apparaissent totalement normaux (SykES, I953 a).

\section{I. - Action des barbiturates.}

Chez la poule, contrairement à ce qui se passe chez le rat, BASTIAN et ZARRow, I952, trouvent que le Nembutal est incapable de bloquer l'excrétion naturelle de L.H. même lorsque la narcose est maintenue durant une période égale à I2 heures (entre Io et 22 heures), heures prévues de sécrétion de L.H. d'après les résultats de ponte des jours antérieurs. (L'intervalle entre 1'ovulation et l'oviposition étant pris égal à 26 heures et l'intervalle entre L.H. et l'ovulation variant de 4 à Io heures).

\section{TABLEAU XI}

Incapacité du Nembutal à bloquer la sécrétion naturelle de L.H. à la dose de 20 à $30 \mathrm{mg}$ toutes les 20 à 30 minutes.

\begin{tabular}{c|c|c}
\hline $\begin{array}{c}\text { Nombre } \\
\text { de poules }\end{array}$ & $\begin{array}{c}\text { Moment de ponte } \\
\text { dans la série }\end{array}$ & $\begin{array}{c}\text { Nombre de poules qui } \\
\text { pondirent l'ouf suivant }\end{array}$ \\
\hline 4 & $\begin{array}{c}\text { Début } \\
\text { Milieu }\end{array}$ & $\begin{array}{c}4 \\
\text { (fin?) }\end{array}$ \\
\hline
\end{tabular}

D'après Bastian et Zarrow, 1952, Proc. Soc. Exp. Biol. Med., 79, 249 (modifié). 
Le Nembutal est également incapable de supprimer la sécrétion de L.H. induite par la progestérone à la dose de I mg par poule en injection intramusculaire.

\section{TABleau XII}

Incapacité du Nembutal à bloquer les ovulations induites par la progestérone.

\begin{tabular}{|c|c|c|c|c|}
\hline Groupe & $\begin{array}{c}\text { Durée anesthésie } \\
\text { en heures }\end{array}$ & $\begin{array}{l}\text { Nombre } \\
\text { de poules }\end{array}$ & ayant ovulé & $\begin{array}{c}\text { Intervalle entre injection } \\
\text { et l'heure de ponte } \\
\text { (en heures) }\end{array}$ \\
\hline $\begin{array}{l}\text { Progestérone seulement... } \\
\text { Progestérone + anesthésie. }\end{array}$ & 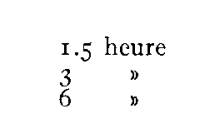 & $\begin{array}{r}12 \\
14 \\
5 \\
13\end{array}$ & $\begin{array}{r}83 \\
100 \\
80 \\
77\end{array}$ & $\begin{array}{l}35, \mathrm{I} \pm 0,9 \\
35,4 \pm \mathrm{I}, 3 \\
34,4 \pm \mathrm{r}, 5 \\
35,6 \pm \mathrm{I}\end{array}$ \\
\hline
\end{tabular}

Travaillant selon des vues parallèles FRAPS et CASE (I953) trouvent une diversité surprenante en réponse aux différents barbiturates.

L'acide diallylbarbiturique (Dial.), 1'éthylisopropylbarbiturate de calcium (probarbital de calcium ou Ipral) et le pentobarbital de sodium (Nembutal) injectés de 12 à $\mathrm{I} 6$ heures avant l'ovulation normale de $\mathrm{C}_{\mathrm{I}}$ induisent une ovulation prématurée chez 11 petit nombre significatif de poules traitées.

\section{TABLEAU XIII}

Nombre d'ovulations prématurées du follicule C.I après administration de barbiturate.

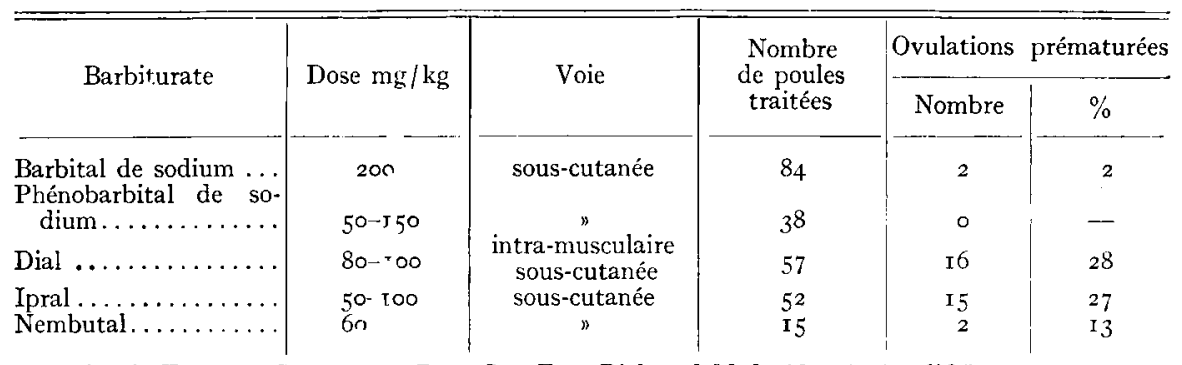

D'après Fraps et Case, 1953, Proc. Soc. Exp. Biol. and Med., 82, I67 (modifié).

De plus l'acide diallylbarbiturique et le Nembutal activent plus qu'ils ne suppriment la tendance de la progestérone à induire l'ovulation à la dose subovulatoire de $0, \mathrm{I} \mathrm{mg}$ par poule.

Il est à remarquer que le phénobarbital de sodium n'induit aucune ovulation prématurée dans les conditions de cette expérience. Comme nous le verrons, son action est inhibitrice et essentiellement modifiée par l'heure d'injection par rapport à l'heure de l'ovulation attendue.

En outre, le phénobarbital de sodium bloque les ovulations du follicule $\mathrm{Cr}_{\mathrm{I}}$ induites par injection soit de progestérone, soit d'acétate de désoxycorticostérone soit de testostérone à la condition que l'injection de ces hormones suive de 30 à 40 minutes 1'administration du barbiturate. 


\section{TABLEAU XIV}

Action synergique de la progestérone et du Dial, de la progestérone et du Nembutal.

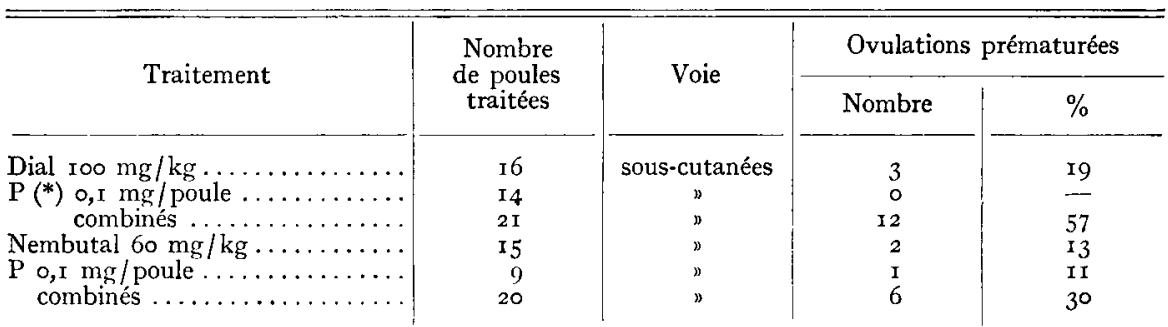

P* Progestérone injectée en sous-cutanée 30 à 40 minutes après administration de barbiturates, d'après FraPS et CASE, I953, Proc. Soc. Exp. Biol. and Med., 82, I I 7 (modifié).

\section{TABLEAU XV}

Blocage par le phénobarbital de sodium des ovulations induites par la progestérone, l'acétate de désoxycorticostérone et la testotérone administrés à la dose de 1 mg par poule.

\begin{tabular}{|c|c|c|c|}
\hline \multirow{2}{*}{ Traitement } & \multirow{2}{*}{$\begin{array}{c}\text { Nombre de poules } \\
\text { traitées }\end{array}$} & \multicolumn{2}{|c|}{ Poules ayant ovulé } \\
\hline & & Nombre & $\%$ \\
\hline 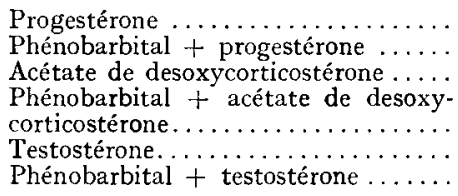 & $\begin{array}{l}19 \\
33 \\
17 \\
22 \\
32 \\
28\end{array}$ & $\begin{array}{r}\text { I } 8 \\
3 \\
\text { I } 5 \\
5 \\
5 \\
\text { I } 3 \\
4\end{array}$ & $\begin{array}{r}95 \\
9 \\
87 \\
23 \\
41 \\
14\end{array}$ \\
\hline
\end{tabular}

D'après FRaPS, Progress in the physiology of farm animals, p. $7 \mathrm{Ix}, 1955$.

Ces nombreuses observations viennent à l'appui de l'hypothèse selon laquelle l'ovulation prématurée accompagnant l'administration de barbiturates agissants, provient d'une excitation neurale, secondaire d'un phénomène de dépression, affectant par un mécanisme humoral la libération de $\mathrm{L}$. $\mathrm{H}$. à partir de l'hypophyse antérieure (FRAPS et CASE, I953); la meilleure preuve étant constituée par la diversité de réponse aux différents barbiturates.

La " composante neurale " incluse directement dans la sécrétion de L.H. réside probablement dans l'hypothalamus, mais l'influence des autres centres peut vraisemblablement jouer un rôle dans la réponse finale de l'hypothalamus lui-même (HARRIS, I952).

\section{II. - Action des agents sympatholytiques et parasympatholytiques.}

$=$ : Des études avec le lapin ont montré que les agents antiadrénergiques de la série haloalkylamine (Dibenamine, SKF 5OI) et certains agents anticholinergiques (atropine et banthine) bloquent le stimulus réflexogène de l'hypophyse s'ils sont injectés par voie intraveineuse quelques fractions de minutes après le coït (SAWYER, MARKEE et col., I949, I95I, I952). Ces agents sont 
également effectifs dans le blocage de l'activation spontanée de l'hypophyse chez les rats cycliques. (EVERETT, I952 ; EVERE'T'T et SAWYER, I950, I953).

Chez la poule ZARRow et BASTIAN (I953) trouvent que le SKF 5OI et le sulfate d'atropine bloquent l'ovulation du follicule CI, que cette ovulation soit naturelle ou induite par la progestérone. L'action de blocage de ces deux produits semble indiquer ici aussi l'existence d'un mécanisme neural à composantes adrénergiques et cholinergiques et que la progestérone agit sur l'hypophyse antérieure par voie nerveuse.

Van Tienhoven et Nalbandov (I954), en employant le premier ceuf (CI) d'une série chez une poule pondant régulièrement, montrent que la dibenamine est capable de bloquer les ovulations spontanées aussi bien que celles induites par la progestérone. Les auteurs en concluent qu'une composante adrénergique est mise en jeu dans les ovulations spontanées aussi bien que provoquées par la progestérone (tableaux XVI et XVII). La dibenamine, bloque beaucoup mieux l'ovulation spontanée lorsque son injection a lieu I4 heures avant 1'ovulation. Son action est progressivement moins efficace à ro, 8, 6 et 4 heures avant l'ovulation avec une anomalie à I2 heures (tanx de blocage plus bas). Le fait que le blocage le plus effectif ait lieu à I4 heures suggère aux auteurs que le stimulus aboutissant à la sécrétion de $\mathrm{L}_{\text {. }}$.H. et à l'ovulation peut avoir lieu environ I4 heures avant la rupture du follicule.

\section{TABLEAU XVI}

Blocage de l'ovulation à l'aide de dibenamine administrée par voie intraveineuse à intervalles variés avant l'ovulation présumée.

\begin{tabular}{|c|c|c|c|c|c|}
\hline $\begin{array}{c}\text { Dose } \\
(\mathrm{mg} / \mathrm{kg})\end{array}$ & $\begin{array}{l}\text { Temps avant l'ovula- } \\
\text { tion attendue (heures) }\end{array}$ & $\begin{array}{l}\text { Nombre } \\
\text { d'oiseaux }\end{array}$ & $\begin{array}{l}\text { Nombre } \\
\text { d'oiseaux } \\
\text { ovulant }\end{array}$ & $\begin{array}{c}\text { Nombre } \\
\text { d'oiseaux } \\
\text { n'ovulant pas }\end{array}$ & $\%$ bloqués \\
\hline 30 & 4 & 7 & 7 & 0 & 0 \\
\hline 30 & 6 & 12 & 9 & 3 & 25 \\
\hline 30 & 8 & I 2 & 6 & 6 & $5^{\circ}$ \\
\hline 30 & IO & I 4 & 6 & 8 & $57, \mathrm{I}$ \\
\hline 30 & I 2 & I6 & I3 & 3 & 18,7 \\
\hline 30 & I 4 & I 6 & 2 & I4 & 87,5 \\
\hline- & 8 & $r_{3}$ & I 2 & $I$ & - \\
\hline
\end{tabular}

D'après Van Tienhoven, Nalbandov et Norton, Endocrinology, 54, p. 605 , I954 (partiel).

\section{TABLEAU XVII}

Effet de la dibénamine et de l'atropine sur le blocage des ovulations induites par la progestérone chez les poules en ponte.

\begin{tabular}{|c|c|c|c|c|c|c|c|c|c|}
\hline \multirow{2}{*}{ Traitement } & \multirow{2}{*}{ Dose } & \multirow{2}{*}{ Voie } & \multirow{2}{*}{$\begin{array}{l}\text { Nombre } \\
\text { d'oiseaux }\end{array}$} & \multicolumn{2}{|c|}{$\begin{array}{c}\text { Ovulation } \\
\text { prématurée }\end{array}$} & \multicolumn{2}{|c|}{$\begin{array}{l}\text { Pas d'ovulation } \\
\text { prématurée }\end{array}$} & \multicolumn{2}{|c|}{ N'ovulèrent pas } \\
\hline & & & & $\mathrm{Nb}$ & $\%$ & $\mathrm{Nb}$ & $\%$ & $\mathrm{Nb}$ & $\%$ \\
\hline Progestérone. & $0,6 \mathrm{mg} / \mathrm{kg}$ & Intravein. & I 4 & I I & 78,5 & 3 & $2 \mathrm{I}, 5$ & o & 0 \\
\hline Progestérone & $0,6 \mathrm{mg} / \mathrm{kg}$ & " & & & & & & & 760 \\
\hline $\begin{array}{r}\text { + Dibenamine. } \\
\text { Progestérone } \\
+ \text { Atropine... }\end{array}$ & $\begin{array}{r}30 \mathrm{mg} / \mathrm{kg} \\
\text { o,6 } \mathrm{mg} / \mathrm{kg} \\
30 \mathrm{mg} / \mathrm{kg}\end{array}$ & " & I7 & I I & 64,7 & 12 & 35,3 & 10 & 29,4 \\
\hline
\end{tabular}

D'après Van Tienhoven, Nalbandov et Norton, Endocrinology, 54, p. 605, I954 (modifié). 


\section{III. - Importance de l'heure d'injection.}

La singularité du phénobarbital de sodium, liée à la constatation que ce produit inhibe de façon effective les ovulations induites par la progestérone lorsqu'il est injecté I4 heures au moins avant l'ovulation $C_{I}$ et $40 \mathrm{p}$. Ioo des ovulations $\mathrm{C}_{2}$ quand il est injecté 9 heures avant l'ovulation $\mathrm{C} 2$, amena FRAPS et CONNER à approfondir son action en fonction de l'heure d'injection par rapport à l'heure d'ovulation attendue (FRAPS, I955) et à la résumer dans le tableau suivant :

\section{TABLEAU XVIII}

Suppression de l'ovulation par le phénobarbital de sodium à la dose de $100 \mathrm{mg} / \mathrm{kg}$.

\begin{tabular}{|c|c|c|c|c|}
\hline \multirow{2}{*}{ Ovulation } & \multirow{2}{*}{$\begin{array}{l}\text { Temps écoulé entre } \\
\text { l'injection et l'heure } \\
\text { d'ovulation attendue }\end{array}$} & \multirow{2}{*}{$\begin{array}{c}\text { Nombres de poules } \\
\text { traitées }\end{array}$} & \multicolumn{2}{|c|}{ Ovulations supprimées } \\
\hline & & & Nombre & $\%$ \\
\hline $\mathrm{Cr}_{\mathrm{I}}$ & $\begin{array}{r}38 \\
34 \\
31 \\
27 \\
22 \\
18 \\
13 \\
9\end{array}$ & $\begin{array}{r}\text { IO } \\
\text { I I } \\
9 \\
7 \\
\text { I I } \\
\text { I2 } \\
\text { IO } \\
\text { IO }\end{array}$ & $\begin{array}{l}9 \\
9 \\
6 \\
6 \\
8 \\
3 \\
0 \\
0\end{array}$ & $\begin{array}{l}90 \\
82 \\
67 \\
86 \\
73 \\
25\end{array}$ \\
\hline $\mathrm{C}_{2}$ & $\begin{array}{r}23 \\
18 \\
17 \\
15 \\
13 \\
11 \\
9 \\
6\end{array}$ & $\begin{array}{r}\text { I I } \\
\text { I I } \\
\text { I4 } \\
9 \\
\text { I } 2 \\
\text { I3 } \\
\text { I } 2 \\
9\end{array}$ & $\begin{array}{r}\text { I I } \\
\text { IO } \\
\text { I } 2 \\
4 \\
6 \\
6 \\
5 \\
\text { I }\end{array}$ & $\begin{array}{r}\text { IOO } \\
9 \mathrm{I} \\
86 \\
44 \\
50 \\
46 \\
42 \\
\text { II }\end{array}$ \\
\hline
\end{tabular}

D'après FraPS, I955, Extrait de Progress in the physiology of farm animals, p. 718.

Cette action du phénobarbital de sodium peut difficilement être associée à une suppression de sécrétion de L.H. puisque, comme nous l'avons vu, la sécrétion de L.H. précède 1'ovulation de 7 à 8 heures (FRAPS, RILEY et OLSEN, I942) ou de 8 à I4 heures (Van Tienhoven, Nalbandov et NorTon, I954). Il semble plus vraisemblable d'admettre, devant sa sélectivité, que le phénobarbital de sodium interrompt ou supprime les processus de maturation folliculaire.

Pout tester cette hypothèse, FrRAPS et CONNER, I954, étudient l'action des barbiturates et d'agents anticholinergiques et antiadrénergiques administrés 38 heures avant 1'ovulation CI attendue.

La signification de ces résultats devient apparente si l'on compare les effets des mêmes agents après administration I4 heures avant l'ovulation attendue c'est-à-dire après une injection destinée à affecter la composante neurale du mécanisme spécifique de libération de L.H. 


\section{'TABLEAU XIX}

Suppression de l'ovulation C1 par les barbiturates et les "agents bloquants" administrés 38 heures avant l'ovulation attendue.

\begin{tabular}{|c|c|c|c|c|c|}
\hline \multirow{2}{*}{ Agent } & \multicolumn{2}{|c|}{ Traitement } & \multirow{2}{*}{$\begin{array}{l}\text { Nombre } \\
\text { de poules } \\
\text { traitces }\end{array}$} & \multicolumn{2}{|c|}{ Ovulations supprimées } \\
\hline & Voie & Dose & & Nombre & $\%$ \\
\hline 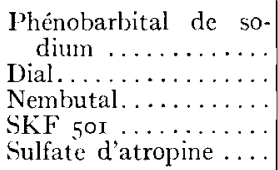 & $\begin{array}{l}\text { Sous-cutanće } \\
\text { Intra-musculaire } \\
\text { Sous-cutance } \\
\text { Intra-musculaire } \\
\text { Intra-péritonćale }\end{array}$ & $\begin{array}{l}100 \mathrm{mg} / \mathrm{kg} \\
80 \mathrm{mg} / \mathrm{kg} \\
60 \mathrm{mg} / \mathrm{kgr} \\
\text { I0 } \mathrm{mgr} / \mathrm{kr} \\
250 \mathrm{mg} / \mathrm{foule}\end{array}$ & $\begin{array}{l}10 \\
10 \\
11 \\
9 \\
9\end{array}$ & $\begin{array}{l}9 \\
8 \\
6 \\
7 \\
6\end{array}$ & $\begin{array}{l}90 \\
80 \\
55 \\
77 \\
67\end{array}$ \\
\hline
\end{tabular}

\section{TABLEAU XX}

Action des barbiturates et des "agents bloquants" $\grave{a}$ des doses identiques, sur le follicule C1 en fonction de l'heure d'injection.

\begin{tabular}{|c|c|c|c|c|c|}
\hline \multirow{2}{*}{ Agents } & \multicolumn{3}{|c|}{ Injection dans les i4 $h$ avant ovulation } & \multicolumn{2}{|c|}{ Injection $3^{8} \mathrm{~h}$ avant ovulation } \\
\hline & $\begin{array}{l}\text { Avancement } \\
\text { ovulation } \%\end{array}$ & $\begin{array}{l}\text { Suppression } \\
\text { ovulation \% }\end{array}$ & Auteurs & $\begin{array}{l}\text { Suppression } \\
\text { ovulation \% }\end{array}$ & Auteurs \\
\hline Phénobarbital. ... & o & & FraPs, Case I953 & 90 & $\begin{array}{c}\text { FRAPS, CONNER } \\
\text { I } 954\end{array}$ \\
\hline Dial...... & $\mathbf{2 8}$ & & ” & 80 & $\begin{array}{c}2934 \\
n\end{array}$ \\
\hline Nembutal....... & I3 & & $"$ & 55 & ${ }^{2}$ \\
\hline Dibénamine...... & & & $\begin{array}{c}\text { Van TIENIOVNeN et } \\
\text { col. I } 954\end{array} \mid$ & $9^{2}$ & FraPS I955 \\
\hline $\mathrm{SKF}_{501} \ldots \ldots \ldots$ & & $\begin{array}{c}30 \text { à } 40 \\
79\end{array}$ & $\begin{array}{c}\text { FraPs et ConNer } \\
\text { I } 954 \\
\text { ZarRow et Bastian }\end{array}$ & 77 & $\begin{array}{c}\text { FRAPS, CONNER } \\
\text { I } 954\end{array}$ \\
\hline Sulfate d'atropine. & & 79 & $\mid \begin{array}{c}\text { I95.3 } \\
\text { ZARROW et BASTIAN } \\
\text { 195.3 }\end{array}$ & 67 & 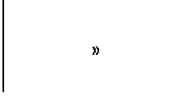 \\
\hline
\end{tabular}

La suppression de l'ovulation $C_{I}$ par les barbiturates et agents bloquants injectés 38 heures avant l'ovulation attendue indique presque certainement le blocage de mécanismes nerveux contrôlant un processus essentiel de la période précédant l'ovulation. I a suppression directe de L.H. paraît en effet improbable. FraPs et CONNER (I954) suggèrent que l'activité nerveuse en question contrôle la sécrétion de 1'hormone (ou hormones) hypophysaire nécessaire au fonctionnement du follicule ovarien en cours de maturation.

Afin de préciser ces résultats et de déterminer la durée de stimulation hypophysaire durant l'induction de l'ovulation à l'aide de la progestérone, Van TIENHOVEN, I955, reprend ces expériences en injectant la dibénamine ou l'atropine à des intervalles de temps croissant à partir de l'injection de progestérone. Des résultats résumés dans le tableau ci-dessous, l'auteur ne retient que ceux obtenus à l'aide de l'atropine, les résultats provenant de l'injection de dibénamine n'étant pas valables à cause des nombreuses atrésies obtenues dans le cas d'injection simultanée de ce produit et de la progestérone. 
TABLEAU XXI

Intensité de blocage des ovulations induites par la progestérone à différents intervalles de temps entre l'injection de progestérone et les injections de dibénamine ou d'atropine.

\begin{tabular}{|c|c|c|c|c|c|c|c|}
\hline $\begin{array}{c}\text { Intervalle } \\
\text { progestérone à } \\
\text { dibénanine }\end{array}$ & $\begin{array}{l}\text { Nombre } \\
\text { d'oiseaux } \\
\text { tratités }\end{array}$ & \multicolumn{2}{|c|}{$\begin{array}{l}\text { Ovulations } \\
\text { prématirées } \\
\text { bloquées }\end{array}$} & $\begin{array}{c}\text { lntervalle } \\
\text { prosestérone at } \\
\text { atropine }\end{array}$ & $\begin{array}{l}\text { Nonubre } \\
\text { d'oiseaux } \\
\text { traités }\end{array}$ & \multicolumn{2}{|c|}{$\begin{array}{l}\text { Ovulations } \\
\text { prématurées } \\
\text { bloquées }\end{array}$} \\
\hline$o$ & 26 & $22(\mathbf{2})$ & 84,6 & 0 & 20 & $17\left({ }^{2}\right)$ & 85,0 \\
\hline $\mathbf{I}$ & I 5 & I $3(2)$ & 86,7 & I & 12 & 8 & 67,7 \\
\hline $\mathrm{I}, \overline{5}$ & $\therefore$ & 一 & $\longrightarrow$ & $\mathrm{I}, 5$ & I 2 & () $\left({ }^{2}\right)$ & $75, \circ$ \\
\hline 2 & - & 一 & - & 2 & I 2 & $6(2)$ & 50,0 \\
\hline 2,5 & $\rightarrow$ & 一 & $\longrightarrow$ & 2,5 & 12 & 4 & $3,3,3$ \\
\hline 3 & I 5 & 9()$\left.^{2}\right)$ & 60,0 & 3 & 13 & 0 & - \\
\hline 4 & - & $\cdots$ & - & 4 & 12 & 0 & 一 \\
\hline 5 & I 4 & $7(1)$ & 50,0 & 5 & II & 2 & I. 8,2 \\
\hline 6 & 14 & 5 & 35,7 & 6 & I I & 2 & $\mathrm{I} 8,2$ \\
\hline Progestérone seule & 19 & 3 & I 5,8 & I'rogestérone seule & $2 \mathrm{I}$ & 2 & 9,5 \\
\hline
\end{tabular}

(1) Différence avec la progestérone seule significative au seuil de $5 \%$.

(2) Différence avec la progestérone seule significative au seuil de $1 \%$.

D'après Van Tienhoven, Endocrinology, 56, 667, 1955.

Van Tienhoven conclut que chez la poule les composantes adrénergiques et cholinergiques, mises en jeu dans la stimulation hypophysaire due à la progestérone, ont des durées sensiblement différentes et que le minimum de temps de stimulation des composantes cholinergiques est au minimum de 26 minutes et au maximum de $2 \mathrm{~h} 30$. Ni l'atropine ni la dibénamine n'interfèrent en effet avec le processus de l'ovulation en soi puisque ni l'un ni l'autre de ces deux produits, injectés en même temps qu'un extrait d'hypophyse antérieure, n'inhibent l'ovulation prématurée ainsi induite.

\section{IV. - Ovulation et Ponte.}

WARREN et SCOTT, I935 ont montré qu'une ovulation ne peut normalement se produire lorsqu'un œuf se trouve dans une quelconque partie de 1'oviducte. Chez une poule pondant tous les jours, 1'ovulation a lieu I4 à 75 minutes après l'oviposition. Mais l'ovulation suivante n'est pas modifiée quand l'œuf se trouvant dans l'utérus est prématurément expulsé par un broyage manuel. De même des injections d'éphédrine qui retardent la ponte de 24 à 36 heures chez certaines poules n'affectent pas l'ovulation de façon visible, ou du moins pas toujours, puisque un second ouf mal formé peut à l'occasion être pondu après la ponte de l'œuf retardé (WEISs et STURKIE, I952).

Réciproquement, l'ablation du follicule ovarien rompu ou l'ablation de ce follicule et du follicule en maturation le plus ancien, au moment où l'ovocyte issu du follicule rompu est dans l'oviducte, provoque presque invariablement sa rétention durant I à 7 jours. L'ablation d'autres parties de l'ovaire à des temps analogues, sans ablation simultanée du follicule le plus récemment rompu, ne provoque pratiquement jamais un maintien comparable de l'œuf se trouvant dans 1'oviducte. I1 semble donc que le follicule rompu de la poule soit un facteur important dans la détermination du moment de la ponte de 
l'œuf formé à partir de l'ovule qu'il contenait précédemment (RothcrrLD et FRAPS, I944),

De même, si l'ovulation est prématurément induite de quelques heures, par exemple 3 à 6 heures avant l'oviposition attendue, il peut se produire que cette oviposition soit également en avance de quelques heures (FRAPS, I942). Il semble donc bien que le follicule ovarien ait une influence prépondérante sur l'heure de la ponte. Rappelons ici que tous les essais pour mettre en évidence la progestérone dans le follicule ovarien du pigeon et de la poule ont échoué, ainsi que la recherche d'une formation correspondant aux " corps jaunes " des mammifères. La progestérone ou une hormone physiologiquement équivalente a cependant été isolée dans le sang des poules en ponte, des poules non en ponte et des coqs, mais non chez les chapons. FrAPS, HOOKER et ForBEs, r948 et 1949 .

\section{C. - ORIGINE DES SERIES}

Dans des conditions normales d'éclairement, l'ovulation d'un ovocyte ne peut se produire que de 6 à I4 heures environ, c'est-à-dire durant 8 heures sur les 24 que comporte une journée. L'ovulation et donc l'excrétion de L.H. sont soumises à un rythme diurne strict, la ponte et l'ovulation associées étant inversées par inversion du rythme d'éclairement.

Diverses hypothèses ont essayé de tenir compte et du retard d'un ovule sur l'autre à l'intérieur d'une série et de la formation des séries. Deux hypothèses se trouvent en présence.

\section{I. - Hypothèse de FRAPS}

Essaie avant tout d'expliquer le retard anormal du follicule Cr de la série à venir sur le follicule $\mathrm{C} n$ de la série écoulée.

S'appuyant sur l'action des barbiturates (existence d'une composante nerveuse) et sur l'action du benzoate d'œstradiol et de L.H. (C2 retardé d'un jour se comporte comme CI) FRAPS, I954, émet une hypothèse selon laquelle les centres nerveux responsables de l'excrétion de L.H. auraient un seuil d'excitation variable en fonction de l'heure de la journée. Pour arriver à cette conclusion, l'auteur est amené à s'appuyer sur un certain nombre d'hypothèses secondaires, à savoir que :

Io Les centres nerveux intéressés dans l'excrétion de L.H. répondent à des taux définis d'une hormone provenant de l'ovaire, plus spécialement du follicule en maturation et appelée " hormone d'excitation ".

$2^{0}$ Le taux sanguin "d'hormone d'excitation "s'accroît avec le temps.

$3^{\circ}$ Dans un cycle de longueur donné chaque excitation précède l'ovulation correspondante d'un intervalle de temps constant. (Évalué à 8 heures sur la base des ovulations induites par la progestérone). Les excitations successives sont alors reportées dans le temps de façon identique aux ovulations.

$4^{\circ}$ Chaque courbe de concentration sanguine de "1'hormone d'excitation" autre que celle correspondant à $\mathrm{CI}$, est retardée dans la journée d'une valeur égale au retard de l'excitation précédente.

Le graphique ci-dessous schématise les relations qui existeraient entre les concentrations "d'hormones d'excitation " et la variation diurne du seuil 
d'excitabilité des composantes nerveuses présidant à l'excrétion de L.H. chez une poule pondant selon un cycle de 7 jours autrement dit par séries de 6 œufs.

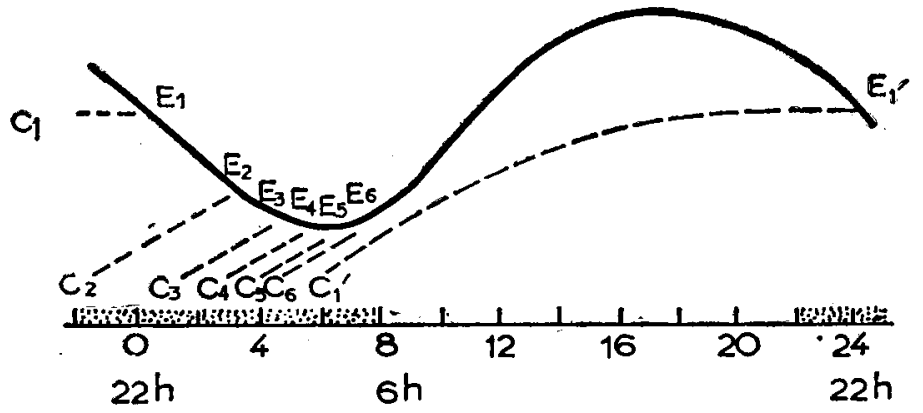

GRAPHIQUE IV. - En abscisse échelle des temps, l'heure $O$ correspondant à 22 heures. Les parties ombrées représentent les heures de nuit. En ordonnée, la concentration d'hormone nécessaire à l'excitation. En trait plein, courbe de variation diurne du seuil d'excitation nerveuse. En pointillé, courbes de concentration sanguine de "l'hormone d'excitation " correspondant aux follicules $\mathrm{Cr}_{1}, \mathrm{C}_{2}$ en fonction du temps.

D'après Fraps R. M., Proc. Nat. Acad. Sci. U. S. A., 40, 348, I954 a.

Un tel schéma a le mérite de tenir compte de nombreuses particularités de la série.

Il peut expliquer en particulier, les relations de temps entre l'heure de ponte des différents oufs d'une même série. L'intervalle de temps qui sépare les œufs diminue en effet au début de la série et augmente vers la fin.

Les phénomènes qui président à la fin d'une série et aut retard de 40 à 44 heures que subit le follicule $\mathrm{Cr}$ de la série suivante. se trouvent également expliqués.

I'hypothèse permet aussi de comprendre les modes d'action du benzoate d'œstradiol et de L.H. tels qu'ils ont été reportés plus haut. En effet, si l'on admet que le benzoate d'œstradiol augmente légèrement le setil de réponse de la composante nerveuse, il devient évident que les follicules $\mathrm{Cr}$ se trouvent moins bloqués que les follicules $\mathrm{C} z$ et que ces derniers puissent ovuler le jour suivant à l'heure habituelle de $\mathrm{Cr}$ avec un pourcentage d'ovulations inhibées égal à celui de $\mathrm{C}_{r}$. Ces observations, donnant une explication simple de l'effet des œstrogènes, constituent la meilleure preuve que le mécanisme nerveux contrôlant l'excrétion de L.H., à partir de l'hypophyse antérieure présente un cycle diurne de son seuil de réponse aux " hormones d'excitation ".

L'action de L.H., inhibant l'ovulation du follicule $\mathrm{C}_{2}$ en la reportant au lendemain matin, peut également s'expliquer si l'on admet avec Fraps et FÉvold, I955 que, dans certaines proportions, L.H. et F.S.H., peuvent favoriser la production d'œstrogènes chez la poule, ce qui nous ramène au cas précédent.

I a théorie de Fraps, revient donc à attribuer un tôle prépondérant au seuil d'excitation du mécanisme de libération de L.H. et non au follicule dont le retard à la maturation semblerait capable à lui seul d'induire ce retard de 40 à 44 heures, retard dont on pourrait simplement rechercher l'origine dans la vitesse de vitellogenèse. C'est dans ce sens que BASTIAN et ZARrow ont émis en 1955, une nouvelle hypothèse. 


\section{II. - Hypothèse de ZARROW et BASTIAN}

Après avoir vérifié l'existence d'un rythme diurne dans le contrôle des mécanismes de la ponte et l'inhibition des mécanismes de rupture du follicule par la lumière et l'activité, ZARROw et BASTIAN, I955, émettent une nouvelle théorie explicative des particularités du cycle de ponte chez la poule : le cycle serait le résultat de l'asynchronisme du rythme diurne et de la maturation plus ou moins rythmique des follicules. Mais pour arriver à cette conclusion les auteurs sont obligés de postuler que le mécanisme aboutissant à la rupture du follicule fonctionne toutes les nuits durant une période assez longue de 8 heures et d'émettre l'hypothèse que les follicules en maturation sont séparés les uns des autres par des intervalles relativement constants à moins que leur ovulation ne soit possible, dans les conditions normales, à des stades de maturité plus ou moins avancée.

Pour ces auteurs, il n'y aurait donc pas incompétence hypophysaire, mais défaut de maturité du follicule. Cette explication paraît également satisfaisante puisque nous avons vu que l'efficacité des injections inductrices d'ovulation dépend essentiellement du degré de maturité du follicule ovarien et que la prématurité du follicule mesurée par sa réponse aux injections, est pour partie fonction de la dose. L'hypothèse tient en outre compte del'abaissement apparent du seuil de réponse du follicule $C_{I}$ à une injection de $\mathcal{L}_{1}$.H., effectuée 24 heures avant l'ovulation naturelle aussi bien que du report, après inhibition temporaire, de l'ovulation du follicule $\mathrm{C} 2$ et de son comportement en follicule $\mathrm{Cr}$.

\section{CONCLUSIONS}

\section{Facteurs hormonaux.}

I.H., provoque l'expulsion du premier globule polaire dans les 4 heures qui suivent son administration et la déhiscence du follicule ovarien dans les 6 à 8 heures. Cet intervalle apparaît constant quelle que soit la place de l'ovulation dans le cycle. A des doses sub ou susoptimales cette gonadotrophine inhibe l'ovulation.

La progestérone, l'acétate de désoxycorticostérone, la testotérone (cette dernière hormone à un degré moindre) induisent également l'ovulation aux doses indiquées. L'action de la désoxycorticostérone et de la progestérone sont à peu près identiques.

Tous les faits (hypophysectomie, prétraitement à l'aide de P.M.S., action des sympatholytiques et parasympatholytiques) démontrent que 1'hypophyse joue un rôle de relais dans l'action de la progestérone qui agirait par voie nerveuse.

Dans des conditions d'application identiques par rapport à l'ovulation à venir, la progestérone et l'hormone lutéinisante ont une action plus nette sur le follicule $\mathrm{Cr}$ que sur le follicule $\mathrm{C} 2$. L'administration de l'une ou de l'autre de ces hormones à des temps optima permet de prolonger les séries de 2 à 3 ovocytes.

Les œstrogènes : benzoate d'œstradiol, dipropionate d'œstradiol et diéthylstilbestrol, aux doses employées, inhibent partiellement 1'ovulation du follicule $\mathrm{C}_{I}$ et celle du follicule $\mathrm{C} 2$, de façon pratiquement indépendante de l'intervalle 
de temps séparant l'injection de l'ovulation attendue (dans les limites d'un intervalle de $4 \mathrm{I}$ heures avant 1'ovulation de $\mathrm{C}_{\mathrm{I}}$ et de 28 heures avant l'ovulation de $\mathrm{C} 2$ ) le follicule $\mathrm{C}_{2}$ étant plus facilement inhibé que le follicule $\mathrm{Cr}_{1}$.

\section{Facteurs nerveux.}

Une légère irritation d'origine traumatique de la paroi du magnum ou de 1'isthme inhibe durant une vingtaine de jours l'ovulation du follicule mûr sans provoquer pour autant l'atrésie des follicules se trouvant sur la grappe ovarienne au moment de l'opération. Une stimulation analogue de l'utérus est sans effet. Le mécanisme a-t-il un rôle physiologique dans la régulation de la ponte bien que l'ovule ne demeure que $4 \mathrm{~h}$. I/2 dans la région sensible? L'hypothèse demande à être développée et vérifiée par de nouveaux travaux.

Action des barbiturates. - Le Nembutal est incapable d'inhiber l'ovulation naturelle ou provoquée par la progestérone comme il le fait chez les mammifères.

L'injection, 38 heures avant l'ovulation de $C_{I}$, de phénobarbital, d'acide diallylbarbiturique ou de pentobarbital de sodium inhibe l'ovulation consécutive. La faible durée d'action de ces barbiturates amène à penser, que dans ce cas, leur effet inhibiteur s'exerce non pas sur la libération de L.H., à partir de l'hypophyse antérieure, mais plutôt sur un des processus de maturation folliculaire. Les deux derniers barbiturates cités ont un effet diamétralement opposé lorsqu'ils sont administrés I4 heures avant l'ovulation attendue. Comme dans le cas des oestrogènes, le follicule $\mathrm{C}_{2}$ est plus sensible que le follicule $\mathrm{C}_{\mathrm{I}}$ à une inhibition provoquée par injection de phénobarbital.

L'acide diallylbarbiturique et le pentobarbital de sodium augmentent l'action inductrice de la progestérone administrée à des doses subovulatoires, le phénobarbital de sodium bloque les ovulations qui seraient normalement induites par cette même hormone.

Action des anticholinergiques et antiadrenergiques. - Le sulfate d'atropine, la dibénamine, le S.K.F. 50I, inhibent l'ovulation naturelle aussi bien que provoquée par la progestérone. De cet ensemble d'expériences effectuées à l'aide des barbiturates et des sympatho et parasympatholytiques se dégage la notion de l'intervention du système nerveux dans la libération, à partir de l'hypophyse antérieure, de la quantité de L.H., nécessaire à l'ovulation. Cette intervention met en jeu des composantes cholinergiques, et adrènergiques ; pour les premières, la durée de stimulation nécessaire à l'ovulation est comprise entre 26 et 150 minutes.

\section{Maturité et activation folliculaire.}

Tout processus aboutissant à une activation partielle du follicule (doses subovulatoires de L.H., interruption du processus d'induction de l'ovulation) provoquent l'atrésie des follicules mis en cause.

Les nombreux résultats obtenus sur le mode d'action de L.H., injectée à des intervalles de temps variables avant l'ovulation attendue, ainsi que 
l'expérience effectuée à l'aide du phénobarbital, dans les mêmes conditions, mettent en valeur la notion de maturité identique des follicules $\mathrm{Cr}_{1}$ et $\mathrm{C}_{2}$ à des temps comparables après l'ovulation précédente. Il devient dès lors difficile d'expliquer l'action des oestrogènes, dont l'effet est indépendant de ce temps, si ce n'est à l'aide de l'hypothèse de Fraps.

Il semble, devant les connaissances actuelles, que les nouveaux travaux doivent s'orienter vers une détermination de la notion de maturité du follicule qui demeure encore assez imprécise dans les dernières 24 heures de son évolution. En particulier, une connaissance plus approfondie des derniers stades de la vitellogenèse et de leur liaison avec l'aptitude du follicule à répondre à une dose définie de L.H., peut seule nous amener à mieux séparer la fin de l'ovogénèse du mécanisme de l'ovulation en vue d'une compréhension plus nette de la fonction ovarienne chez la poule.

\section{RÉFÉRENCES BIBLIOGRAPHIQUES}

BASTIAN (J. W.) and Zarrow (M. X.). - Failure of Nembutal to block ovulation in the hen. Proc. Soc. Exp. Biol. Med., 79, p. 249, I952.

Bastian (J. W.) and Zarrow (M. X.). - New hypothesis for the asynchronous ovulatory cycle of the domestic hen (gallus domesticus). Poult. Sci. 34, p. 776, I955.

BATES (R. W.), LAHR (E. L.) and RIDDLE (O.). - The gross action of prolactin and follicle-stimulating hormone on the mature ovary and sex accessories fowl. American Phys., 111, p. 36r, I935.

Dauzier (L.), Ortavant (R.), Thubault (C.) et Wintenberger (S.). Recherches expérimentales sur le rôle de la progestérone dans le cycle sexuel de la brebis et de la chèvre. Annales Endocrinol., 14, p. 553, I953.

EDGAR (D. G.). - The progestérone content of body fluids and tissues. The Journal of endocrinology, 10, p. 54, I953.

EVERETT (J. W.). - Presumptive hypothalamic control of spontaneous ovulation. Ciba foundation colloquia on endocrinology IV, p. I76, I952.

EVERETT (J. W.) and SAWYER (C. H.). - A 24-hour periodcity in the "L. H.release apparatus " of female rats, disclosed by barbiturate sedation. Endocrinology, 47, p. I98, I950.

EVERETT (J. W.) and SAWYER (C. H.). - Estimated duration of the spontaneous activation which causes release of ovulation hormone from the rat hypophysis. Endocrinology, 52, p. 83, I953.

FEVOLD (H. L.). - Annals N. Y. Acad. Sci., 43, p. 32 I, I943.

FRAPS (R. M.). - Synchronized induction of ovulation and premature oviposition in the domestic fowl. Anat. Rec., 84, p. 52I, I942 (abstracts).

FRAPS (R. M.). - Differential ovulatory reaction of first and subsequent follicle of the hen's clutch. Anat. Rec., 96, p. 573, I946 (abstracts).

Fraps (R. M.). - Neural basis of diurnal periodicity in release of ovulationinducing hormone in fowl. Proc. Nat. Acad. Sci. U. S. A., 40, p. 348, I954a.

FRAPS (R. M.). - Timing functions of a neural component in the mechanism of release of pituitary gonadotrophin for ovulation in the hen. Tenth World's Poultry Congress. Section Papers, p. I70, I54 $b$.

FRAPS (R. M.). - - Progress in the physiology of farm animals. Butterworths publications. London, r955.

FRAPS (R. M.). - The varying effects of sex hormones in birds. Memoirs of the society for endocrinology $n^{\circ} 4$, I955.

FraPs (R. M.) and CASE (J. F.). - Premature ovulation in domestic fowl following administration of certain barbiturates. Proc. Soc. Exp. Biol. Med. 82, p. I67, I953. 
Fraps (R. M.). and ConNer (M. H.). - Neurohypophysial control of follicular maturation in the domestic fowl. Nature, 1\%4, p. II48, I954.

FRAPS (R. M.) and DURY (A.). - Relative sensivity to certain ovulation inducing agents of first and subsequent follicles of clutch sequences in the hen. Anat. Rec. 84, p. 453, I942 a (abstracts).

FraPs (R. M.) and DURy (A.). - The occurence of regression in ovarien follicles of the hen following the administration of a luteinizing extract. Anat. Rec. 84, p. 52I, I942 $b$ (abstracts).

FRAPS (R. M.) and DURY (A.). - Occurrence of premature ovulation in the domestic fowl following administration of progesterone. Proc. Soc. Exp. Biol. Med., 52, p. 346, I943.

FraPs (R. M.), FEVOLD (H. L.) and NEHER (B. H.). - Ovulatory response of the hen to presumptive luteinizing and other fractions free from fowl anterior pituitary tissue. Anat. Rec., 99, p. 57 I, I047 (abstracts).

FRAPS (R. M.) and FEVOLD (H. L.). - Delaying action of gonadotrophins on ovulation in the hen. Proc. Soc. Exp. Biol. Med., 90, p. 440, I955.

Fraps (R. M.), Hooker (C. W.) and Forbes (T. R.). - Progestérone in blood plasma of the ovulating hen. Science, 108, p. 86, I948.

Fraps (R. M.), Hooker (C. W.) and Fories ('l'. R.). - Progestérone in blood plasma of cocks and non ovulating hens. Science, 109, 493, I949.

Fraps (R. M.), OlsFN (M. W.) and NehER (B. H.). - Forced ovulation of normal ovarian follicles in the domestic fowl. Proc. Soc. Exp. Biol. Med., 50, p. 308, I942.

FRAPS (R. M.) and RILEY (G. M.). - Hormone-induced ovulation in the domestic fow1. Proc. Soc. Exp. Biol. Med., 49, p. 253, I942.

Fraps (R. M.), Riley (G. M.) and Oisen (M. W.). - Time required for induction of ovulation following intravenous injection of hormone preparation in fowl. Proc. Soc. Exp. Biol. Med., 50, p. 313, I942.

Harris (G. W.). - Hypothalamic control of the anterior pituitary gland. Ciba Foundation Coll. Endocri. 4, p. I06, I952.

Huston (T. M.) and Nalbandov (A. V.). - Neurohumoral control of the pituitary in the fowl. Endocrinology, 52,, p. I49, I953.

MAuger (H. M.). - The autonomic innervation of the female genitalia in the domestic fowl and its correlation with the aortic branchings. Am. J. Vet. Res., 2, p. 447, I94I.

Nalbandov (A.) and CARD (L. E.). - Effect of F.S.H. and L. H. upon the ovaries of immature chicks and low-producing hens. Endocrinology, 38, p. 7I, I946.

NEHER (B. H.) and FRAPS (R. M.). - The addition of eggs to the hen's clutch by repeated injections of ovulation-inducing hormones. Endocrinology, 46, p. 482, I950.

OLSEN (M. W.) and FRAPS (R. M.). - Maturation changes in the hen's ovum. J. Exp. Zool., 114, p. 475, I950.

OLSEN (M. W.), Fraps (R. M.) and NEHER (B. H.). - Timing of normal and experimental induced preovulatory changes in the hen's ovum. Anat. Rec., 99, p. 660, 1947.

RILEY (G. M.) and FRAPS (R. M.). - Biological assays of the male chicken pituitary for gonadotrophin potency. Endocrinology, 30, p. 529, I942.

ROTHCHIL (I.). - The time of release of ovulating hormone from the anterior pituitary gland of the domestic hen. Anat. Rec., 96, p. 542, I946.

ROTHCHILD (I.) and FRAPS (R. M.). - On the function of the ruptured ovarian follicle of the domestic fowl. Proc. Soc. Exper. Biol. Med., 56, p. 79, I944.

RoTHCHILD (I.) and Fraps (R. M.). - The effect of hypophysectomy on the ovulability of the ovarian follicle of the domestic hen. J. Clin. Endocrinol., 8, p. 6I5, I948.

RoTHCHILD (I.) and FRAPS (R. M.). - The interval between normal release of 
ovulating hormone and ovulation in the domestic hen. Endocrinology, 44, p. I34, I949 $a$.

ROTHCHILD (I.) and FRAPS (R. M.). - The induction of ovulating hormone relesase from the pituitary of domestic hen by means of progestérone Endocrinology, 44, p. I4I, I949 b.

Sawyer (C. H.), Markee (J. E.) and Everett (J. W.). - Failure of Nerve blocking agents to prevent the effects of placental gonadotrophins in the rabbit. Endocrinology, 49, p. I62, I95I.

Sawyer (C. H.), Markee (J. E.) and Townsend (B. F.). - Cholinergic and adrenergic components in the neuro humoral control of the release of L. H. in the rabbit. Endocrinology, 44, p. I8, I949.

Shaffiner (C. S.). - Progesterone induced molt. Poult. Sci., 34, p. 840, 1955.

Sturkie (P. D.). - Avian Physiology. Comstock publishing associates Ythaca New York, I954.

Sykes (A. M.). - Some observations on oviposition in the fowl. Quart. J. Exp. Physiol., 38, p. 6I, I953a.

Sykes (A. M.). Premature oviposition in the hen. Nature, I72, p. I098, I953 $b$.

VAN DER MEULEN (J. B.). - Determination de la mue et de 1'ovulation par les hormones. $7^{\text {me }}$ Congrès Mondial d'aviculture Section I.

Van Tienhoven (A.). - Further study on the netrogenic blockade of $\mathrm{L}_{1}$. H. release in the hen. Anat. Rec., 115, p. 374, I953.

VAN TIEnhoven (A.). - Duration of stimulation of the hen's hypophysis in progestérone induced ovulation. Anat. Rec., 118, p. 364, I 954 (abstracts).

VAN TIENHOVEN (A.). - The duration of stimulation of the fowl's anterior pituitary for progesterone induced L. H. release. Endocrinology, 56, 667 , I955.

VAN Tienhoven (A.), Nalbandov (A. V.) and Norton (H. W.). - Effect of Dibenamine on progesterone induced and "spontaneous " ovulation in the hen. Endocrinology, 54, p. 605, I954.

WARren (D. C.) and ScotT (H. M.). - - The time factor in egg formation. Poult. Sci., 14, p. I95, I935.

WeISS (H. S.) and STURKIE (P. D.). - Time of oviposition as affected by neuromimetic drugs. Poult. Sci., 31, p. 227, 1952.

Zarrow (M. X.) and BASTIAN (J. W.). - Blockade of ovulation in the hen with adrenolytic and parasympatholytic drugs. Proc. Soc. Exp. Biol. Med., 84, p. 457, I953.

ZARrow (M. X.), HisAw (F. L.) and Bryans (F.). - Conversion of desoxycorticosterone acetate to progesterone in vivo. Endocrinology, 46, p. $403,1950$.

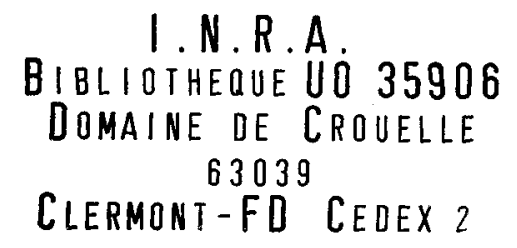

